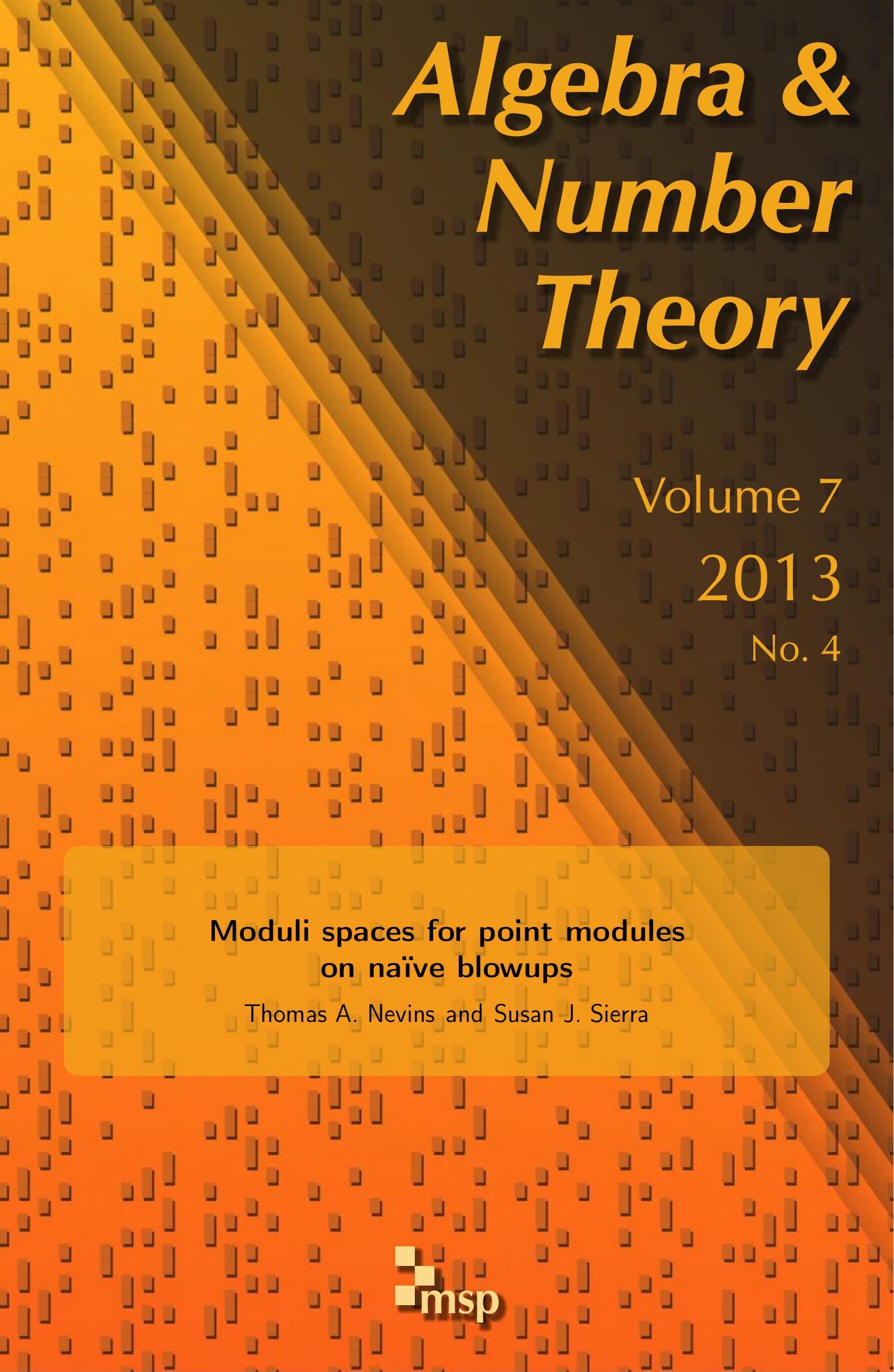




\title{
Moduli spaces for point modules on naïve blowups
}

\author{
Thomas A. Nevins and Susan J. Sierra
}

\begin{abstract}
The naïve blowup algebras developed by Keeler, Rogalski, and Stafford, after examples of Rogalski, are the first known class of connected graded algebras that are noetherian but not strongly noetherian. This failure of the strong noetherian property is intimately related to the failure of the point modules over such algebras to behave well in families: puzzlingly, there is no fine moduli scheme for such modules although point modules correspond bijectively with the points of a projective variety $X$. We give a geometric structure to this bijection and prove that the variety $X$ is a coarse moduli space for point modules. We also describe the natural moduli stack $X_{\infty}$ for embedded point modules — an analog of a "Hilbert scheme of one point" - as an infinite blowup of $X$ and establish good properties of $X_{\infty}$. The natural map $X_{\infty} \rightarrow X$ is thus a kind of "Hilbert-Chow morphism of one point" for the naïve blowup algebra.
\end{abstract}

\section{Introduction}

One of the important achievements of noncommutative projective geometry is the classification of noncommutative projective planes, such as the three-dimensional Sklyanin algebra $\mathrm{Skl}_{3}$, by Artin, Tate, and Van den Bergh [Artin et al. 1990]. More formally, these are Artin-Schelter regular algebras of dimension 3, noncommutative graded rings that are close analogs of a commutative polynomial ring in three variables; see [Stafford and Van den Bergh 2001] for a discussion. The key method of [Artin et al. 1990] is to study point modules, that is, cyclic graded modules with the Hilbert series of a point in projective space. Given a noncommutative projective plane $R$, the authors describe a moduli scheme for its point modules. This allows them to construct a homomorphism from $R$ to a well understood ring, providing a first step in describing the structure of the noncommutative plane itself.

The techniques described above work in a more general context. Let $\mathbb{k}$ be an algebraically closed field; we assume $\mathbb{k}$ is uncountable although for some of the results quoted this hypothesis is unnecessary. A $\mathbb{k}$-algebra $R$ is said to be strongly noetherian if, for any commutative noetherian $\mathbb{k}$-algebra $C$, the tensor

MSC2010: primary 16S38; secondary 16D70, 16W50, 14A20, 14D22.

Keywords: naïve blowup, point module, point space. 
product $R \otimes_{\mathbb{k}} C$ is again noetherian. By a general result of Artin and Zhang [2001, Theorem E4.3], if $R$ is a strongly noetherian $\mathbb{N}$-graded $\mathbb{k}$-algebra, then its point modules are parametrized by a projective scheme. Rogalski and Zhang [2008] used this result to extend the method of [Artin et al. 1990] to strongly noetherian connected graded $\mathbb{k}$-algebras that are generated in degree 1 . (An $\mathbb{N}$-graded $\mathbb{k}$ algebra $R$ is connected graded if $R_{0}=\mathbb{k}$.) Their method constructs a map from the algebra to a twisted homogeneous coordinate ring (see Section 2 for definitions) on the scheme $X$ parametrizing point modules. For example, Sklyanin algebras are strongly noetherian, and here $X$ is an elliptic curve. The homomorphism here gives the well known embedding of an elliptic curve in a noncommutative $\mathbb{P}^{2}$.

Although it was believed for a time that all connected graded noetherian algebras would be strongly noetherian, Rogalski [2004] showed this was not the case. His example was generalized in joint work with Keeler and Stafford [Keeler et al. 2005; Rogalski and Stafford 2007] to give a geometric construction of a beautiful class of noncommutative graded algebras, known as naïve blowups, that are noetherian but not strongly noetherian. Along the way, they showed that point modules for naïve blowups - viewed as objects of noncommutative projective geometry in a way we make precise below - cannot behave well in families: there is no fine moduli scheme of finite type for such modules.

In the present paper, we systematically develop the moduli theory of point modules for the naïve blowups $S$ of [Keeler et al. 2005; Rogalski and Stafford 2007]. Roughly speaking, we show that there is an analog of a "Hilbert scheme of one point on $\operatorname{Proj}(S)$ " that is an infinite blowup of a projective variety. This infinite blowup is quasicompact and noetherian as an fpqc-algebraic stack (a notion we make precise in Section 4). Furthermore, we show there is a coarse "moduli space for one point on $\operatorname{Proj}(S)$ " - it is, in fact, the projective variety from which the naïve blowup was constructed. These are the first descriptions in the literature of moduli structures for point modules on a naïve blowup.

More precisely, let $X$ be a projective $\mathbb{k}$-variety of dimension at least 2, let $\sigma$ be an automorphism of $X$, and let $\mathscr{L}$ be a $\sigma$-ample (see Section 2) invertible sheaf on $X$. We follow the standard convention that $\mathscr{L}^{\sigma}:=\sigma^{*} \mathscr{L}$. Let $P \in X$ (in the body of the paper, we let $P$ be any zero-dimensional subscheme of $X$ ), and assume that the $\sigma$-orbit of $P$ is critically dense; that is, it is infinite and every infinite subset is Zariski dense. For $n \geq 0$, let

$$
\mathscr{I}_{n}:=\mathscr{I}_{P}{\Phi_{P}^{\sigma}}_{P} \cdots \mathscr{I}_{P}^{\sigma^{n-1}} \quad \text { and } \quad \mathscr{L}_{n}:=\mathscr{L} \otimes \mathscr{L}^{\sigma} \otimes \cdots \otimes \mathscr{L}^{\sigma^{n-1}}
$$

Define $\mathscr{Y}_{n}:=\mathscr{I}_{n} \otimes \mathscr{L}_{n}$, and let

$$
S:=S(X, \mathscr{L}, \sigma, P):=\bigoplus_{n \geq 0} H^{0}\left(X, \mathscr{Y}_{n}\right)
$$


The algebra $S$ is the naïve blowup associated to the data $(X, \mathscr{L}, \sigma, P)$.

If $\mathscr{L}$ is sufficiently ample, then $S$ is generated in degree 1 ; alternatively, a sufficiently large Veronese of $S$ is always generated in degree 1 . We will assume throughout that $S$ is generated in degree 1 .

A point module is a graded cyclic $S$-module $M$ with Hilbert series $1+t+t^{2}+\cdots$. We say $M$ is an embedded point module if we are given, in addition, a surjection $S \rightarrow M$ of graded modules. Two embedded point modules $M$ and $M^{\prime}$ are isomorphic if there is an $S$-module isomorphism from $M$ to $M^{\prime}$ that intertwines the maps from $S$.

We begin by constructing a moduli stack for embedded point modules. Recall that $X_{\infty}$ is a fine moduli space (or stack) for embedded point modules if there is an $S$-module quotient $S \otimes_{\mathbb{k}} \mathrm{O}_{X_{\infty}} \rightarrow M$ that is a universal family for point modules; that is, $M$ is an $X_{\infty}$-flat family of embedded $S$-point modules with the property that if $S \otimes_{\mathbb{k}} C \rightarrow M^{\prime}$ is any $C$-flat family of embedded point modules for a commutative $\mathbb{k}$ algebra $C$, then there is a morphism $\operatorname{Spec}(C) \stackrel{f}{\rightarrow} X_{\infty}$ and an isomorphism $f^{*} M \cong M^{\prime}$ of families of embedded $S$-point modules. Let $X_{n}$ be the blowup of $X$ at $\Phi_{n}$; there is an inverse system $\cdots \rightarrow X_{n} \rightarrow X_{n-1} \rightarrow \cdots \rightarrow X$ of schemes. Let $X_{\infty}:=\lim _{\longleftarrow} X_{n}$. This inverse limit exists as a stack. More precisely, in Definition 4.1, we introduce the notion of an fpqc-algebraic stack. We then have:

Theorem 1.1. The inverse limit $X_{\infty}$ is a noetherian fpqc-algebraic stack. The morphism $X_{\infty} \rightarrow X$ is quasicompact. Moreover, $X_{\infty}$ is a fine moduli space for embedded S-point modules.

We have been told that similar results were known long ago to M. Artin; however, they seem not to have been very widely known even among experts, nor do they seem to have appeared in the literature.

Note that the stack $X_{\infty}$ is discrete: its points have no stabilizers. Thus, $X_{\infty}$ is actually a $\mathbb{k}$-space in the terminology of [Laumon and Moret-Bailly 2000]; in particular, this justifies our use of the phrase "fine moduli space" in the statement of the theorem. However, $X_{\infty}$ does not seem to have an étale cover by a scheme and hence does not have the right to be called an algebraic space.

We recall that, by definition, the noncommutative projective scheme associated to $S$ is the quotient category Qgr- $S=$ Gr- $S$ /Tors- $S$ of graded right $S$-modules by the full subcategory of locally bounded modules. A point object in Qgr-S is the image of (a shift of) a point module. If $S$ is a commutative graded algebra generated in degree 1, Qgr- $S$ is equivalent to the category of quasicoherent sheaves on $\operatorname{Proj}(S)$; this justifies thinking of Qgr- $S$ as the noncommutative analog of a projective scheme.

If $R$ is strongly noetherian and generated in degree 1, then a result of Artin and Stafford [Keeler et al. 2005, Theorem 10.2] shows that point objects of Qgr- $R$ are parametrized by the same projective scheme $X$ that parametrizes embedded point 
modules. On the other hand, for naïve blowups $S=S(X, \mathscr{L}, \sigma, P)$ as above, we have:

Theorem 1.2 [Keeler et al. 2005, Theorem 1.1]. The algebra $S$ is noetherian but not strongly noetherian. Moreover, there is no fine moduli scheme of finite type over $\mathbb{k}$ parametrizing point objects of $\mathrm{Qgr}-\mathrm{S}$.

By contrast, [Keeler et al. 2005] gives a simple classification (that fails in families), namely that point objects are in bijective correspondence with points of $X$ : to a point $x \in X$, we associate the $S$-module $\bigoplus H^{0}\left(X, \mathbb{k}_{x} \otimes \mathscr{L}_{n}\right)$. In the present paper, we explain how these two facts about point objects of Qgr- $S$ naturally fit together.

Assume that $\mathscr{L}$ is sufficiently ample (in the body of the paper, we work with any $\sigma$-ample $\mathscr{L}$ by considering shifts of point modules). Let $F$ be the moduli functor of embedded point modules over $S$. Define an equivalence relation $\sim$ on $F(C)$ by saying that $M \sim N$ if their images are isomorphic in $\operatorname{Qgr}-S \otimes_{\mathbb{k}} C$. We obtain a functor $G$ : Affine schemes $\rightarrow$ Sets by sheafifying (in the fpqc topology) the presheaf $G^{\text {pre }}$ of sets defined by $\operatorname{Spec} C \mapsto F(C) / \sim$.

A scheme $Y$ is a coarse moduli scheme for point objects if it corepresents the functor $G$; that is, there is a natural transformation $G \rightarrow \operatorname{Hom}_{\mathbb{k}}(\cdot, Y)$ that is universal for natural transformations from $G$ to schemes.

Our main result is the following:

Theorem 1.3. The variety $X$ is a coarse moduli scheme for point objects in Qgr-S.

This gives a geometric structure to the bijection discovered by Keeler, Rogalski, and Stafford.

Corollary 1.4. There is a fine moduli space $X_{\infty}$ for embedded $S$-point modules but only a coarse moduli scheme X for point objects of Qgr-S.

It may be helpful to compare the phenomenon described by Corollary 1.4 to a related, though quite different, commutative phenomenon. Namely, let $Y$ be a smooth projective (commutative) surface. Fix $n \geq 1$. Let $R=\mathbb{C}[Y]$ denote a homogeneous coordinate ring of $Y$ (associated to a sufficiently ample invertible sheaf on $Y$ ), and consider graded quotient modules $R \rightarrow M$ such that $\operatorname{dim} M_{l}=n$ for $l \gg 0$. By a general theorem of Serre, the moduli space for such quotients is the Hilbert scheme of $n$ points on $Y$, denoted $\operatorname{Hilb}^{n}(Y)$. This is a smooth projective variety of dimension $2 n$. Alternatively, remembering only the corresponding objects $[M]$ of Qgr- $R \simeq \mathrm{Q} \operatorname{coh}(Y)$ and imposing the further $S$-equivalence relation [Huybrechts and Lehn 1997, Example 4.3.6], we get the moduli space $\operatorname{Sym}^{n}(Y)$ for semistable length- $n$ sheaves on $Y$, which equals the $n$-th symmetric product of $Y$. The latter moduli space is only a coarse moduli space for semistable sheaves. One has the Hilbert-Chow morphism $\operatorname{Hilb}^{n}(Y) \rightarrow \operatorname{Sym}^{n}(Y)$, which is defined by taking a quotient $R \rightarrow M$ to the equivalence class of $M$. It is perhaps helpful to view the 
moduli spaces and map $X_{\infty} \rightarrow X$ associated to the algebra $S$ in light of the theorems stated above, that is, as a kind of "noncommutative Hilbert-Chow morphism of one point" for a naïve blowup algebra $S(X, \mathscr{L}, \sigma, P)$.

In work in preparation, we generalize the results in [Rogalski and Zhang 2008] by proving a converse, of sorts, to Theorem 1.3. Namely, suppose $R$ is a connected graded noetherian algebra generated in degree 1 , that $R$ has a fine moduli space $X_{\infty}$ for embedded point modules, that $R$ has a projective coarse moduli scheme $X$ for point objects of Qgr- $R$, and that the spaces $X_{\infty}$ and $X$ and the morphism $X_{\infty} \rightarrow X$ between them have geometric properties similar to those of the spaces we encounter in the theorems above. Then, we show, there exist an automorphism $\sigma$ of $X$, a zero-dimensional subscheme $P \subset X$ supported on points with critically dense orbits, an ample and $\sigma$-ample invertible sheaf $\mathscr{L}$ on $X$, and a homomorphism $\phi$ : $R \rightarrow S(X, \mathscr{L}, \sigma, P)$ from $R$ to the naïve blowup associated to this data; furthermore, $\phi$ is surjective in large degree. This construction gives a new tool for analyzing the structure of rings that are noetherian but not strongly noetherian. Details will appear in [Nevins and Sierra 2012].

\section{Background}

In this section, we give needed definitions and background. We begin by discussing bimodule algebras: this is the correct way to think of the sheaves $\mathscr{S}_{n}$ defined above. Most of the material in this section was developed in [Van den Bergh 1996; Artin and Van den Bergh 1990], and we refer the reader there for references. Our presentation follows that in [Keeler et al. 2005; Sierra 2011].

Convention 2.1. Throughout the paper, by variety (over $\mathbb{k}$ ), we mean an integral separated scheme of finite type over $\mathbb{k}$.

Throughout this section, let $\mathbb{k}$ be an algebraically closed field and let $A$ denote an affine noetherian $\mathbb{k}$-scheme, which we think of as a base scheme.

Definition 2.2. Let $X$ be a scheme of finite type over $A$. An $O_{X}$-bimodule is a quasicoherent $O_{X \times X}$-module $\mathscr{F}_{F}$ such that, for every coherent submodule $\mathscr{F}^{\prime} \subseteq \mathscr{F}_{\text {}}$, the projection maps $p_{1}, p_{2}: \operatorname{Supp} \mathscr{F}^{\prime} \rightarrow X$ are both finite morphisms. The left and right $\mathrm{O}_{X}$-module structures associated to an $\mathrm{O}_{X}$-bimodule $\mathscr{F}$ are defined respectively as $\left(p_{1}\right)_{*} \mathscr{F}$ and $\left(p_{2}\right)_{*} \mathscr{F}$. We make the notational convention that when we refer to an $O_{X}$-bimodule simply as an $O_{X}$-module, we are using the left-handed structure (for example, when we refer to the global sections or higher cohomology of an $\mathrm{O}_{X}$-bimodule). All $\mathrm{O}_{X}$-bimodules are assumed to be $\mathrm{O}_{A}$-symmetric.

There is a tensor product operation on the category of bimodules that has the expected properties [Van den Bergh 1996, Section 2].

All the bimodules that we consider will be constructed from bimodules of the following form: 
Definition 2.3. Let $X$ be a projective scheme over $A$, and let $\sigma, \tau \in \operatorname{Aut}_{A}(X)$. Let $(\sigma, \tau)$ denote the map

$$
X \rightarrow X \times_{A} X \quad \text { defined by } \quad x \mapsto(\sigma(x), \tau(x)) .
$$

If $\mathscr{F}_{F}$ is a quasicoherent sheaf on $X$, we define the $\mathscr{O}_{X}$-bimodule ${ }_{\sigma} \mathscr{F}_{\tau}:=(\sigma, \tau)_{*} \mathscr{F}$. If $\sigma=1$ is the identity, we will often omit it; thus, we write $\mathscr{F}_{\tau}$ for ${ }_{1} \mathscr{F}_{\tau}$ and $\mathscr{F}_{F}$ for the $\mathscr{O}_{X}$-bimodule ${ }_{1} \mathscr{F}_{1}=\Delta_{*} \mathscr{F}$, where $\Delta: X \rightarrow X \times{ }_{A} X$ is the diagonal.

Definition 2.4. Let $X$ be a projective scheme over $A$. An $O_{X}$-bimodule algebra, or simply a bimodule algebra, $\mathscr{B}$ is an algebra object in the category of bimodules. That is, there are a unit map $1: \mathscr{O}_{X} \rightarrow \mathscr{B}$ and a product map $\mu: \mathscr{B} \otimes \mathscr{B} \rightarrow \mathscr{B}$ that have the usual properties.

We follow [Keeler et al. 2005] and define the following:

Definition 2.5. Let $X$ be a projective scheme over $A$, and let $\sigma \in \operatorname{Aut}_{A}(X)$. A bimodule algebra $\mathscr{B}$ is a graded $\left(O_{X}, \sigma\right)$-bimodule algebra if

(1) there are coherent sheaves $\mathscr{P}_{n}$ on $X$ such that $\mathscr{B}=\bigoplus_{n \in \mathbb{Z} 1}\left(\mathscr{B}_{n}\right)_{\sigma^{n}}$,

(2) $\mathscr{B}_{0}=\mathscr{O}_{X}$, and

(3) the multiplication map $\mu$ is given by $\mathrm{O}_{X}$-module maps $\mathscr{B}_{n} \otimes \mathscr{B}_{m}^{\sigma^{n}} \rightarrow \mathscr{B}_{n+m}$, satisfying the obvious associativity conditions.

Definition 2.6. Let $X$ be a projective scheme over $A$, and let $\sigma \in \operatorname{Aut}_{A}(X)$. Let $\mathscr{R}=\bigoplus_{n \in \mathbb{Z}}\left(\mathscr{R}_{n}\right)_{\sigma^{n}}$ be a graded $\left(O_{X}, \sigma\right)$-bimodule algebra. A right $\mathscr{R}$-module $\mathcal{M}$ is a quasicoherent $\mathscr{O}_{X}$-module $\mu$ together with a right $\mathfrak{O}_{X}$-module map $\mu: M \otimes \mathscr{R} \rightarrow M$ satisfying the usual axioms. We say that $M$ is graded if there is a direct sum decomposition $\mathcal{M}=\bigoplus_{n \in \mathbb{Z}}\left(\mathcal{M}_{n}\right)_{\sigma^{n}}$ with multiplication giving a family of $O_{X}$-module maps $\mathcal{M}_{n} \otimes \mathscr{R}_{m}^{\sigma^{n}} \rightarrow \mathcal{M}_{n+m}$ obeying the appropriate axioms.

We say that $\mathcal{M}$ is coherent if there are a coherent $O_{X}$-module $\mathcal{M}^{\prime}$ and a surjective map $\mathcal{M}^{\prime} \otimes \mathscr{R} \rightarrow \mathcal{M}$ of ungraded $\mathscr{R}$-modules. We make similar definitions for left $\mathscr{R}$-modules. The bimodule algebra $\mathscr{R}$ is right (left) noetherian if every right (left) ideal of $\mathscr{R}$ is coherent. A graded $\left(\mathrm{O}_{X}, \sigma\right)$-bimodule algebra is right (left) noetherian if and only if every graded right (left) ideal is coherent.

We recall here some standard notation for module categories over rings and bimodule algebras. Let $C$ be a commutative ring, and let $R$ be an $\mathbb{N}$-graded $C$ algebra. We define Gr- $R$ to be the category of $\mathbb{Z}$-graded right $R$-modules; morphisms in Gr- $R$ preserve degree. Let Tors- $R$ be the full subcategory of modules that are direct limits of right bounded modules. This is a Serre subcategory of Gr- $R$, so we may form the quotient category

$$
\text { Qgr- } R:=\text { Gr- } R / \text { Tors }-R \text {. }
$$


(We refer the reader to [Gabriel 1962] as a reference for the category theory used here.) There is a canonical quotient functor from Gr- $R$ to Qgr- $R$.

We make similar definitions on the left. Further, throughout this paper, we adopt the convention that if Xyz is a category, then xyz is the full subcategory of noetherian objects. Thus, we have gr- $R$ and qgr- $R, R$-qgr, etc. If $X$ is a scheme, $\mathrm{O}_{X}$-Mod and $\mathrm{O}_{X}$-mod will denote the categories of quasicoherent and coherent sheaves on $X$, respectively.

Given a module $M \in$ gr- $R$, we define $M[n]:=\bigoplus_{i \in \mathbb{Z}} M[n]_{i}$, where $M[n]_{i}=M_{n+i}$.

For a graded $\left(\mathscr{O}_{X}, \sigma\right)$-bimodule algebra $\mathscr{R}$, we likewise define Gr- $\mathscr{R}$ and gr- $\mathscr{R}$. The full subcategory Tors- $\mathscr{R}$ of Gr- $\mathscr{R}$ consists of direct limits of modules that are coherent as $\mathscr{O}_{X}$-modules, and we similarly define Qgr- $\mathscr{R}:=\mathrm{Gr}-\mathscr{R} /$ Tors- $\mathscr{R}$. We define qgr- $\mathscr{R}$ in the obvious way.

If $\mathscr{R}$ is an $\mathscr{O}_{X}$-bimodule algebra, its global sections $H^{0}(X, \mathscr{R})$ inherit an $\mathscr{O}_{A^{-}}$ algebra structure. We call $H^{0}(X, \mathscr{R})$ the section algebra of $\mathscr{R}$. If $\mathscr{R}=\bigoplus\left(\mathscr{R}_{n}\right)_{\sigma^{n}}$ is a graded $\left(\mathcal{O}_{X}, \sigma\right)$-bimodule algebra, then multiplication on $H^{0}(X, \mathscr{R})$ is induced from the maps

$$
H^{0}\left(X, \mathscr{R}_{n}\right) \otimes_{A} H^{0}\left(X, \mathscr{R}_{m}\right) \stackrel{1 \otimes \sigma^{n}}{\longrightarrow} H^{0}\left(X, \mathscr{R}_{n}\right) \otimes_{A} H^{0}\left(X, \mathscr{R}_{m}^{\sigma^{n}}\right) \stackrel{\mu}{\rightarrow} H^{0}\left(X, \mathscr{R}_{n+m}\right) .
$$

If $\mathcal{M}$ is a graded right $\mathscr{R}$-module, then $H^{0}(X, \mathcal{M})=\bigoplus_{n \in \mathbb{Z}} H^{0}\left(X, \mathcal{M}_{n}\right)$ is a right $H^{0}(X, \mathscr{R})$-module in the obvious way; thus, $H^{0}(X, \cdot)$ is a functor from Gr- $\mathscr{R}$ to $\operatorname{Gr}-H^{0}(X, \mathscr{R})$.

If $R=H^{0}(X, \mathscr{R})$ and $M$ is a graded right $R$-module, define $M \otimes_{R} \mathscr{R}$ to be the sheaf associated to the presheaf $V \mapsto M \otimes_{R} \mathscr{R}(V)$. This is a graded right $\mathscr{R}$-module, and the functor $\cdot \otimes_{R} \mathscr{R}: \mathrm{Gr}-R \rightarrow \mathrm{Gr}-\mathscr{R}$ is a right adjoint to $H^{0}(X, \cdot)$.

The following is a relative version of a standard definition:

Definition 2.7. Let $A$ be an affine $\mathbb{k}$-scheme, and let $q: X \rightarrow A$ be a projective morphism. Let $\sigma \in \operatorname{Aut}_{A}(X)$, and let $\left\{\mathscr{R}_{n}\right\}_{n \in \mathbb{N}}$ be a sequence of coherent sheaves on $X$. The sequence of bimodules $\left\{\left(\mathscr{R}_{n}\right)_{\sigma^{n}}\right\}_{n \in \mathbb{N}}$ is right ample if, for any coherent $\mathrm{O}_{X}$-module $\mathscr{F}_{\mathscr{F}}$, the following properties hold:

(1) $\mathscr{F} \otimes \mathscr{R}_{n}$ is globally generated for $n \gg 0$ (the natural map $q^{*} q_{*}\left(\mathscr{F} \otimes \mathscr{R}_{n}\right) \rightarrow \mathscr{F} \otimes \mathscr{R}_{n}$ is surjective for $n \gg 0$ ) and

(2) $R^{i} q_{*}\left(\mathscr{F} \otimes \mathscr{R}_{n}\right)=0$ for $n \gg 0$ and $i \geq 1$.

The sequence $\left\{\left(\mathscr{R}_{n}\right)_{\sigma^{n}}\right\}_{n \in \mathbb{N}}$ is left ample if, for any coherent $O_{X}$-module $\mathscr{F}$, the following properties hold:

(1) the natural map $q^{*} q_{*}\left(\mathscr{R}_{n} \otimes \mathscr{F} \sigma^{n}\right) \rightarrow \mathscr{R}_{n} \otimes \mathscr{F} \sigma^{n}$ is surjective for $n \gg 0$ and

(2) $R^{i} q_{*}\left(\mathscr{R}_{n} \otimes \mathscr{F}^{\sigma^{n}}\right)=0$ for $n \gg 0$ and $i \geq 1$. 
If $A=\mathbb{k}$, we say that an invertible sheaf $\mathscr{L}$ on $X$ is $\sigma$-ample if the $\mathscr{O}_{X}$-bimodules

$$
\left\{\left(\mathscr{L}_{n}\right)_{\sigma^{n}}\right\}_{n \in \mathbb{N}}=\left\{\mathscr{L}_{\sigma}^{\otimes n}\right\}_{n \in \mathbb{N}}
$$

form a right ample sequence. By [Keeler 2000, Theorem 1.2], this is true if and only if the $\mathcal{O}_{X}$-bimodules $\left\{\left(\mathscr{L}_{n}\right)_{\sigma^{n}}\right\}_{n \in \mathbb{N}}$ form a left ample sequence.

The following result is a special case of a result due to Van den Bergh [1996, Theorem 5.2] although we follow the presentation of [Keeler et al. 2005, Theorem 2.12]:

Theorem 2.8 (Van den Bergh). Let $X$ be a projective $\mathbb{k}$-scheme, and let $\sigma$ be an automorphism of $X$. Let $\mathscr{R}=\bigoplus\left(\mathscr{R}_{n}\right)_{\sigma^{n}}$ be a right noetherian graded $\left(\mathscr{O}_{X}, \sigma\right)$ bimodule algebra such that the bimodules $\left\{\left(\mathscr{R}_{n}\right)_{\sigma^{n}}\right\}$ form a right ample sequence. Then $R=H^{0}(X, \mathscr{R})$ is also right noetherian, and the functors $H^{0}(X, \cdot)$ and $\cdot \otimes_{R} \mathscr{R}$ induce an equivalence of categories qgr- $\mathscr{R} \simeq$ qgr- $R$.

Castelnuovo-Mumford regularity is a useful tool for measuring ampleness and studying ample sequences. We will need to use relative Castelnuovo-Mumford regularity; we review the relevant background here. In the next three results, let $X$ be a projective $\mathbb{k}$-scheme, and let $A$ be a noetherian $\mathbb{k}$-scheme. Let $X_{A}:=X \times A$, and let $p: X_{A} \rightarrow X$ and $q: X_{A} \rightarrow A$ be the projection maps.

Fix a very ample invertible sheaf $O_{X}(1)$ on $X$. Let $O_{X_{A}}(1):=p^{*} \mathscr{O}_{X}(1)$; note $\mathcal{O}_{X_{A}}(1)$ is relatively ample for $q: X_{A} \rightarrow A$. If $\mathscr{F}$ is a coherent sheaf on $X_{A}$ and $n \in \mathbb{Z}$, let $\mathscr{F}(n):=\mathscr{F} \otimes_{X_{A}} O_{X_{A}}(1)^{\otimes n}$. We say $\mathscr{F}$ is $m$-regular with respect to $\mathcal{O}_{X_{A}}(1)$, or just $m$-regular, if $R^{i} q_{*} \mathscr{F}(m-i)=0$ for all $i>0$. Since $O_{X_{A}}(1)$ is relatively ample, $\mathscr{F}$ is $m$-regular for some $m$. The regularity of $\mathscr{F}$ is the minimal $m$ for which $\mathscr{F}$ is $m$-regular; we write it reg $(\mathscr{F})$.

Castelnuovo-Mumford regularity is usually defined only for $\mathbb{k}$-schemes, so we will spend a bit of space on the technicalities of working over a more general base. First note:

Lemma 2.9. Let $\mathscr{F}$ be a coherent sheaf on $X$. Then $\operatorname{reg}(\mathscr{F})=\operatorname{reg}\left(p^{*} \mathscr{F}\right)$.

The fundamental result on Castelnuovo-Mumford regularity is due to Mumford. Theorem 2.10 [Lazarsfeld 2004, Example 1.8.24]. Let $\mathscr{F}$ be an $m$-regular coherent sheaf on $X_{A}$. Then for every $n \geq 0$,

(1) $\mathscr{F}$ is $(m+n)$-regular;

(2) $\mathscr{F}(m+n)$ is generated by its global sections; that is, the natural map

$$
q^{*} q_{*} \mathscr{F}(m+n) \rightarrow \mathscr{F}(m+n)
$$

is surjective;

(3) the natural map $q_{*} \mathscr{F}(m) \otimes_{A} q_{*} \mathrm{O}_{X_{A}}(n) \rightarrow q_{*} \mathscr{F}(m+n)$ is surjective. 
Lemma 2.11. For any 0-regular invertible sheaf $\mathscr{H}$ on $X_{A}$ and any A-point $y$ of $X$, the natural map $q_{*} \mathscr{H} \stackrel{\alpha}{\rightarrow} q_{*}\left(\mathscr{O}_{y} \otimes_{X_{A}} \mathscr{H}\right)$ is surjective.

Proof. This is standard, but we check the details. Since cohomology commutes with flat base change, it suffices to consider the case that $A=\operatorname{Spec} C$, where $C$ is a local ring. Then for any $n \in \mathbb{Z}$, we may consider $\mathcal{O}_{y} \otimes_{X_{A}} \mathscr{H}(n)$ as an invertible sheaf on $A$. Since $C$ is local, as a $C$-module, this is isomorphic to $C$.

We thus have $q_{*}\left(\mathscr{O}_{y} \otimes_{X_{A}} \mathscr{H}\right) \cong C$. Let $I:=\operatorname{Im}(\alpha)$; this is an ideal of $C$.

Let $n \geq 0$, and consider the natural maps

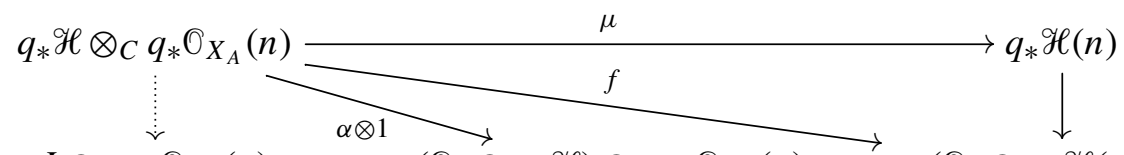

$I \otimes_{C} q_{*} \mathrm{O}_{X_{A}}(n) \longrightarrow q_{*}\left(\mathrm{O}_{y} \otimes_{X_{A}} \mathscr{H}\right) \otimes_{C} q_{*} \mathrm{O}_{X_{A}}(n) \longrightarrow q_{*}\left(\mathrm{O}_{y} \otimes_{X_{A}} \mathscr{H}(n)\right) \cong C$

This diagram clearly commutes, and $\alpha \otimes 1$ factors through $I \otimes_{C} q_{*} O_{X_{A}}(n)$ by construction. Thus, $\operatorname{Im} f \subseteq I$ for all $n$.

On the other hand, by Theorem 2.10(3), $\mu$ is surjective. As $O_{X_{A}}(1)$ is relatively ample, for $n \gg 0$, the right-hand vertical map is surjective. Thus, $f$ is surjective for $n \gg 0$, and so $I=C$.

Let $Z$ be a closed subscheme of $X_{A}$. We say that $Z$ has relative dimension $\leq d$ if, for all $x \in A$, the fiber $q^{-1}(x)$ has dimension $\leq d$ as a $\mathbb{k}(x)$-scheme.

The following is a relative version of Proposition 2.7 of [Keeler 2010]:

Proposition 2.13. Let $X$ be a projective $\mathbb{k}$-scheme. There exists a constant $D$, depending only on $X$ and on $O_{X}(1)$, so that the following holds: for any noetherian $\mathbb{k}$-scheme $A$ and for any coherent sheaves $\mathscr{F}, G$ on $X_{A}$ such that the closed subscheme of $X_{A}$ where $F_{F}$ and $\varphi$ both fail to be locally free has relative dimension $\leq 2$, we have

$$
\operatorname{reg}\left(\mathscr{F} \otimes_{X_{A}} \mathscr{G}\right) \leq \operatorname{reg}(\mathscr{F})+\operatorname{reg}(\mathscr{G})+D
$$

Proof. The statement is local on the base, so we may assume without loss of generality that $A=\operatorname{Spec} C$ is affine. Since standard results such as Theorem 2.10 and Lemma 2.11 hold in this relative context, we may repeat the proof of [Keeler 2010, Proposition 2.7]. The relative dimension assumption ensures the vanishing of $R q_{*}$ that is needed in the proof.

To end the introduction, we define naïve blowups: these are the algebras and bimodule algebras that we will work with throughout the paper. Let $X$ be a projective k-variety. Let $\sigma \in \operatorname{Aut}_{\mathbb{k}}(X)$, and let $\mathscr{L}$ be a $\sigma$-ample invertible sheaf on $X$. Let $P$ be a zero-dimensional subscheme of $X$. We define ideal sheaves

$$
\Phi_{n}:=\Phi_{P} \Phi_{P}^{\sigma} \ldots \mathscr{I}_{P}^{\sigma^{n-1}}
$$


for $n \geq 0$. Then we define a bimodule algebra $\mathscr{S}(X, \mathscr{L}, \sigma, P):=\bigoplus_{n \geq 0}\left(\mathscr{Y}_{n}\right)_{\sigma^{n}}$, where $\mathscr{S}_{n}:=\mathscr{I}_{n} \mathscr{L}_{n}$. Define $S(X, \mathscr{L}, \sigma, P):=H^{0}(X, \mathscr{Y}(X, \mathscr{L}, \sigma, P))$.

Theorem 2.14 [Rogalski and Stafford 2007, Theorems 1.2 and 3.1]. Let $X$ be a projective $\mathbb{k}$-variety with $\operatorname{dim} X \geq 2$. Let $\sigma \in \operatorname{Aut}_{\mathbb{k}}(X)$, and let $\mathscr{L}$ be a $\sigma$-ample invertible sheaf on $X$. Let $P$ be a zero-dimensional subscheme of $X$, and let $\mathscr{S}:=\mathscr{S}(X, \mathscr{L}, \sigma, P)$ and $S:=S(X, \mathscr{L}, \sigma, P)$.

If all points in $P$ have critically dense $\sigma$-orbits, then the following hold:

(1) The sequence of bimodules $\left\{\left(\mathscr{S}_{n}\right)_{\sigma^{n}}\right\}$ is a left and right ample sequence.

(2) $S$ and $\mathscr{S}$ are left and right noetherian, the categories qgr- $S$ and qgr- $\mathscr{Y}$ are equivalent via the global sections functor. Likewise, $S$-qgr and $\mathscr{S}$-qgr are equivalent.

(3) The isomorphism classes of simple objects in qgr- $S \simeq$ qgr- $\mathscr{Y}$ are in one-to-one correspondence with the closed points of $X$, where $x \in X$ corresponds to the $\mathscr{Y}$ module $\bigoplus \mathbb{k}_{x} \otimes \mathscr{L}_{n}$. However, the simple objects in qgr-S are not parametrized by any scheme of finite type over $\mathbb{k}$.

For technical reasons, we will want to assume that our naïve blowup algebra $S$ is generated in degree 1. By [Rogalski and Stafford 2007, Propositions 3.18 and 3.19], this will always be true if we either replace $S$ by a sufficiently large Veronese or replace $\mathscr{L}$ by a sufficiently ample line bundle (for example, if $\mathscr{L}$ is ample, by a sufficiently high tensor power of $\mathscr{L}$ ). If $S$ is generated in degree 1, then by [Rogalski and Stafford 2007, Corollary 4.11], the simple objects in qgr-S are the images of shifts of point modules.

\section{Blowing up arbitrary zero-dimensional schemes}

For the rest of the paper, let $\mathbb{k}$ be an uncountable algebraically closed field. Let $X$ be a projective variety over $\mathbb{k}$, let $\sigma \in \operatorname{Aut}_{\mathbb{k}}(X)$, and let $\mathscr{L}$ be a $\sigma$-ample invertible sheaf on $X$. Let $P$ be a zero-dimensional subscheme of $X$ supported at points with dense (later, critically dense) orbits. Let $\mathscr{Y}:=\mathscr{S}(X, \mathscr{L}, \sigma, P)$, and let $S:=S(X, \mathscr{L}, \sigma, P)$. In this paper, we compare three objects: the scheme parametrizing length- $n$ truncated point modules over $S$, the scheme parametrizing length- $n$ truncated point modules over $\mathscr{S}$, and the blowup of $X$ at the ideal sheaf $\mathscr{I}_{n}=\mathscr{I}_{P} \ldots \mathscr{I}_{P}^{\sigma^{n-1}}$. In this section, we focus on the blowup of $X$. We first give some general lemmas on blowing up the defining ideals of zero-dimensional schemes. These are elementary, but we give proofs for completeness.

Suppose that $X$ is a variety and that $f: Y \rightarrow X$ is a surjective, projective morphism of schemes. Let $\eta$ be the generic point of $X$. We define

$$
Y^{o}:=\overline{f^{-1}(\eta)}
$$


and refer to $Y^{\circ}$, by abuse of terminology, as the relevant component of $Y$. In our situation, $f$ will always be generically one-to-one and $Y^{o}$ will be irreducible with $\left.f\right|_{Y^{o}}$ birational onto its image.

Lemma 3.1. Let $A$ be a variety of dimension $\geq 2$. Let $\mathscr{I}$ be the ideal sheaf of a zero-dimensional subscheme of $A$, and let $\pi: X \rightarrow A$ be the blowup of $A$ at $\Im$. Let $W$ be the scheme parametrizing colength-1 ideals inside $\Im$. Let $\phi: W \rightarrow A$ be the canonical morphism that sends an ideal $\mathscr{F}$ to the support of $\mathscr{I} / \mathscr{F}$. Then there is a closed immersion $c: X \rightarrow W$ that gives an isomorphism between $X$ and $W^{o}$. Further, the following diagram commutes:

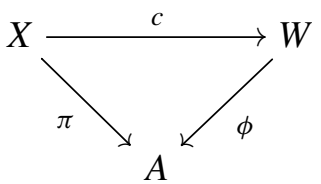

Proof. Without loss of generality, $A=\operatorname{Spec} C$ is affine; let $I:=\mathscr{I}(A)$. We may identify $W$ with Proj $\operatorname{Sym}_{C}(I)$ [Kleiman 1990, Proposition 2.2]; under this identification, $\phi: W \rightarrow A$ is induced by the inclusion $C \hookrightarrow \operatorname{Sym}_{C}(I)$. There is a canonical surjective map of graded $C$-algebras $\operatorname{Sym}_{C}(I) \rightarrow C \oplus \bigoplus_{n \geq 1} I^{n}$, which is the identity on $C$. This induces a closed immersion $c: X \rightarrow W$ with $\phi c=\pi$ as claimed. Further, both $\pi: X \rightarrow A$ and $\phi: W \rightarrow A$ are isomorphisms away from Cosupp $\mathscr{~}$. Thus, $c$ gives a birational closed immersion (and therefore an isomorphism) onto $W^{o}$.

Lemma 3.2. Let $A$ be a variety of dimension $\geq 2$, and let $\Phi$ and $\mathscr{I}$ be ideal sheaves on $A$. Let $\mathscr{Y}:=\Phi$ g. Define $i: X \rightarrow A$ to be the blowup of $A$ at $\mathscr{I}, j: Y \rightarrow A$ to be the blowup of $A$ at $\mathscr{F}$, and $k: Z \rightarrow A$ to be the blowup of $A$ at $\mathscr{K}$.

(a) There are morphisms $\xi: Z \rightarrow X$ and $\omega: Z \rightarrow Y$ so that the diagram

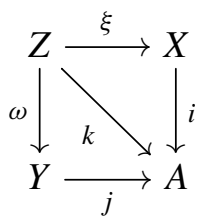

commutes.

(b) We have $Z \cong\left(X \times_{A} Y\right)^{o}$.

(c) Let $W$ be the moduli scheme of subsheaves of $\mathscr{K}$ of colength 1 , and let $V$ be the moduli scheme of subsheaves of $\Phi$ of colength 1 . Let $c: Z \rightarrow W$ and $d: X \rightarrow V$ be the maps from Lemma 3.1, and let $Z^{\prime}:=c(Z)$ and $X^{\prime}:=d(X)$. Then the map $\xi^{\prime}: Z^{\prime} \rightarrow X^{\prime}$ induced from $\xi$ sends $\mathscr{K}^{\prime} \subset \mathscr{K}$ to $\left(\mathscr{K}^{\prime}: \mathscr{g}\right) \cap \mathscr{I}$.

Proof. (a) Since $\xi^{-1}(\mathscr{K}) \mathcal{O}_{Z}=\xi^{-1}(\mathscr{I}) \xi^{-1}(\mathscr{F}) \mathcal{O}_{Z}$ is invertible, the inverse images of both $\mathscr{I}$ and $\mathscr{F}$ on $Z$ are invertible. By the universal property of blowing up 
[Hartshorne 1977, Proposition 7.14], the morphisms $\xi: Z \rightarrow X$ and $\omega: Z \rightarrow Y$ exist and commute as claimed.

For (b) and (c), we may without loss of generality assume $A=\operatorname{Spec} C$ is affine. (b) Let $U:=\left(X \times{ }_{A} Y\right)^{o}$. Let $A^{\prime}:=A \backslash \operatorname{Cosupp} \mathscr{K}$. Then $U$ is the closure of $A^{\prime}$ in $\mathbb{P}_{A}^{n} \times_{A} \mathbb{P}_{A}^{m}$ for appropriate $n$ and $m$.

Let $\phi: \mathbb{P}_{A}^{n} \times_{A} \mathbb{P}_{A}^{m} \rightarrow \Sigma_{n, m} \subset \mathbb{P}_{A}^{n m+m+n}$ be the Segre embedding. Note that the canonical embeddings $Z \subseteq W \subseteq \mathbb{P}_{A}^{n m+m+n}$ actually have $W \subseteq \Sigma_{n, m}$. Since $\phi^{\prime}:=\left.\phi\right|_{U}$ is the identity over $A^{\prime}$ and $Z \subseteq \Sigma_{n, m}$ is the closure of $A^{\prime}$ in $\mathbb{P}_{A}^{n m+n+m}$, we have $\phi^{\prime}(U)=Z$.

Let $p: X \times{ }_{A} Y \rightarrow X$ and $q: X \times_{A} Y \rightarrow Y$ be the projection maps. From the commutative diagram in (a), we obtain a morphism $r: Z \rightarrow X \times_{A} Y$ with $q r=\omega$ and $p r=\xi$. Further, $r$ restricts to $\left(\phi^{\prime}\right)^{-1}$ over $A^{\prime}$. Thus, $r(Z)=U$, and $\phi^{\prime}: U \rightarrow Z$ is an isomorphism.

(c) A point $(x, y) \in \mathbb{P}_{A}^{n} \times{ }_{A} \mathbb{P}_{A}^{m}$ corresponds to a pair of linear ideals $\mathfrak{n} \subset C\left[x_{0}, \ldots, x_{n}\right]$ and $\mathfrak{m} \subset C\left[y_{0}, \ldots, y_{m}\right]$. Let $C\left[\left(x_{i} y_{j}\right)_{i, j}\right] \subset C\left[\left(x_{i}\right)_{i}\right]\left[\left(y_{j}\right)_{j}\right]$ be the homogeneous coordinate ring of $\Sigma_{n, m}$. It is clear the ideal defining $\phi(x, y)=\{x\} \times \mathbb{P}^{m} \cap \mathbb{P}^{n} \times\{y\}$ in $C\left[x_{i} y_{j}\right]$ is generated by $\mathfrak{n}_{1} \cdot\left(y_{0}, \ldots, y_{m}\right)+\left(x_{0}, \ldots, x_{n}\right) \cdot \mathfrak{m}_{1}$.

Let $(x, y) \in\left(X \times_{A} Y\right)^{o}$, where $x$ corresponds to the colength-1 ideal $\mathscr{I}^{\prime} \subseteq \mathscr{I}$ and $y$ corresponds to $\mathscr{F}^{\prime} \subseteq \mathscr{F}$. That $\mathscr{I}^{\prime} \mathscr{F}+\mathscr{I} \mathscr{F}^{\prime}$ gives the ideal $\mathscr{K}^{\prime} \subset \mathscr{K}$ corresponding to $\phi(x, y)$ follows from the previous paragraph together with the fact that the isomorphism $\phi^{\prime}$ between $\left(X \times{ }_{A} Y\right)^{o}$ and $Z$ is given by the Segre embedding.

Since $\phi^{\prime}$ is an isomorphism, any ideal $\mathscr{K}^{\prime}$ corresponding to a point $z \in Z$ may be written $\mathscr{K}^{\prime}=\mathscr{I}^{\prime} \mathscr{F}+\mathscr{I} \mathscr{F}^{\prime}$ for appropriate $\mathscr{I}^{\prime}$ and $\mathscr{F}^{\prime}$. We thus have $\mathscr{I}^{\prime} \subseteq\left(\mathscr{K}^{\prime}: \mathscr{F}\right) \cap \mathscr{I} \varsubsetneqq \mathscr{I}$. Since $\mathscr{I}^{\prime}$ is colength-1, this implies that $\mathscr{I}^{\prime}=\left(\mathscr{K}^{\prime}: \mathscr{F}\right) \cap \mathscr{I}$ as claimed.

Corollary 3.3. Let $X$ be a projective variety of dimension $\geq 2$, let $\sigma \in \operatorname{Aut}_{\mathbb{k}}(X)$, and let $\Phi$ be an ideal sheaf on $X$. Let $\Phi_{n}:=\Phi \Phi^{\sigma} \ldots \oiint^{\sigma^{n-1}}$. For all $n \geq 0$, let $a_{n}: X_{n} \rightarrow X$ be the blowup of $X$ at $\Phi_{n}$. Then there are birational morphisms $\alpha_{n}: X_{n} \rightarrow X_{n-1}$ (for $n \geq 1$ ) and $\beta_{n}: X_{n} \rightarrow X_{n-1}$ (for $n \geq 2$ ) so that the diagrams
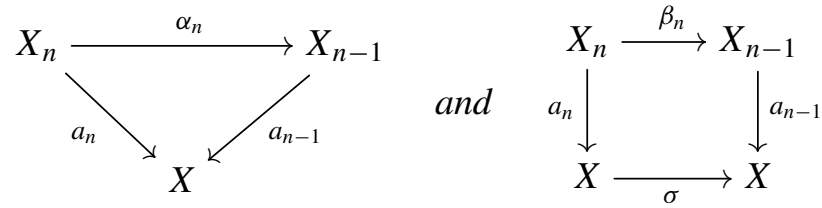

commute.

Proof. Let $\mathscr{K}:=\Phi_{n}$, and let $\zeta: X_{n-1}^{\prime} \rightarrow X$ be the blowup of $X$ at $\Phi_{n-1}^{\sigma}$. Since $\left(\mathscr{I}_{p}\right)^{\sigma} \cong \mathscr{I}_{\sigma^{-1}(p)}$, there is an isomorphism $\theta: X_{n-1}^{\prime} \rightarrow X_{n-1}$ so that the following 
diagram commutes:

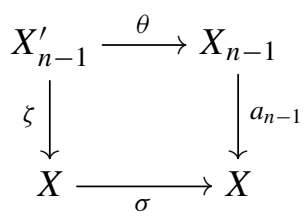

Apply Lemma 3.2(a) with $\mathscr{I}=\Phi_{n-1}^{\sigma}$ and $\mathscr{g}=\Phi_{1}$. We obtain a morphism $\gamma: X_{n} \rightarrow X_{n-1}^{\prime}$ so that

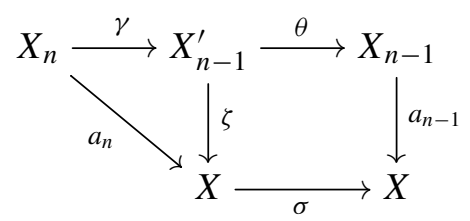

commutes. Let $\beta_{n}:=\theta \gamma: X_{n} \rightarrow X_{n-1}$.

Let $\alpha_{n}$ be the morphism $X_{n} \rightarrow X_{n-1}$ given by Lemma 3.2(a) with $\mathscr{I}=\Phi_{n-1}$ and $\mathscr{g}=g_{1}^{\sigma^{n-1}}$. The diagram

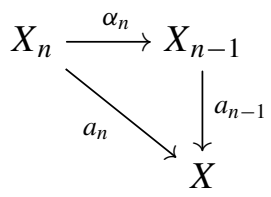

commutes as required.

We will frequently suppress the subscripts on the maps $\alpha_{n}, a_{n}$, etc., when the source and target are indicated. Note that the equation $a_{n}=\alpha_{1} \circ \cdots \circ \alpha_{n}$ that follows from Corollary 3.3 may be written more compactly as $a=\alpha^{n}: X_{n} \rightarrow X$.

\section{Infinite blowups}

In this section, we prove some general properties of infinite blowups that will be useful when we consider moduli spaces of embedded point modules. Such infinite blowups can be handled in two ways: either as pro-objects in the category of schemes or as stacks via the (inverse) limits of such pro-objects in the category of spaces or of stacks. We've chosen to treat infinite blowups as the limits rather than as pro-objects. This is formally the correct choice in the sense that the limit formally contains less information than the pro-object. We note that in our setting, we could also work with the pro-object with no difficulties; however, we have found the language of stacks more natural.

We begin with some technical preliminaries on schemes and stacks. We will work with stacks in the fpqc (fidèlement plat et quasicompact) topology; the fpqc topology of schemes is discussed in [Vistoli 2005, Section 2.3.2]. We are interested 
in a class of stacks that are apparently not algebraic but for which a certain amount of algebraic geometry is still possible. More precisely, recall that a stack $\mathscr{X}$ is called algebraic if the diagonal morphism of $\mathscr{X}$ is representable, separated, and quasicompact; and it has an fppf atlas $f: Z \rightarrow \mathscr{X}$ that is a scheme; that is, $f$ is representable, faithfully flat, and finitely presented. By Artin's theorem [Laumon and Moret-Bailly 2000, Théorème 10.1], the second condition is equivalent to requiring the existence of a smooth, surjective, and representable $f$.

Our stacks are very similar to algebraic stacks, but it seems not to be possible to find a finite-type $f$ for which $Z$ is a scheme. On the other hand, we can find $f$ for which $Z$ is a scheme and $f$ is fpqc — and even formally étale — so in some sense our stacks are the fpqc analogs of algebraic stacks.

Definition 4.1. We will refer to a stack $\mathscr{X}$ for which the diagonal $\Delta: \mathscr{X} \rightarrow \mathscr{X} \times_{\mathbb{k}} \mathscr{X}$ is representable, separated, and quasicompact, and which admits a representable fpqc morphism $Z \rightarrow \mathscr{X}$ from a scheme $Z$, as fpqc-algebraic.

Note that "separated" and "quasicompact" make sense for fpqc stacks by [EGA IV.2 1965, Proposition 2.7.1 and Corollaire 2.6.4]. Unfortunately, in this weaker setting, there are fewer notions of algebraic geometry that one can check fpqclocally and hence fewer adjectives that one can sensibly apply to fpqc-algebraic stacks. Still, one can make sense, for example, of representable morphisms being separated, quasiseparated, locally of finite type or of finite presentation, proper, closed immersions, affine, etc., by [EGA IV.2 1965, Proposition 2.7.1].

Recall [EGA IV.4 1967, Définition 17.1.1] that a morphism of schemes $f: X \rightarrow Y$ is formally étale if, for every affine scheme $Y^{\prime}$, closed subscheme $Y_{0}^{\prime} \subset Y^{\prime}$ defined by a nilpotent ideal, and morphism $Y^{\prime} \rightarrow Y$, the map $\operatorname{Hom}_{Y}\left(Y^{\prime}, X\right) \rightarrow \operatorname{Hom}_{Y}\left(Y_{0}^{\prime}, X\right)$ is bijective. By faithfully flat descent [Vistoli 2005], the definition extends immediately to stacks in the étale, fppf, and fpqc topologies of schemes.

We will say that an fpqc-algebraic stack $\mathscr{X}$ is noetherian if it admits an fpqc atlas $Z \rightarrow \mathscr{X}$ by a noetherian scheme $Z$. Unfortunately, since fpqc morphisms need not be of finite type even locally, it does not seem to be possible to check this property on an arbitrary atlas $Y \rightarrow \mathscr{X}$.

Suppose we have a sequence of schemes $\left\{X_{n} \mid n \in \mathbb{N}\right\}$ and projective morphisms $\pi_{n}: X_{n} \rightarrow X_{n-1}$. We define the infinite blowup $X_{\infty}$ to be the presheaf of sets $X_{\infty}=\lim _{\leftarrow} X_{n}$ : more precisely, let $h_{X_{\infty}}:$ Schemes $^{\text {op }} \rightarrow$ Sets be the functor of points whose value on a scheme $A$ is

$$
h_{X_{\infty}}(A)=\left\{\left(\zeta_{n}: A \rightarrow X_{n}\right)_{n \in \mathbb{N}} \mid \pi_{n} \zeta_{n}=\zeta_{n-1}\right\} .
$$

For each $n$, there is an induced map $\pi: X_{\infty} \rightarrow X_{n}$, where the target space $X_{n}$ is indicated explicitly. 
Proposition 4.2. Suppose that $X:=X_{0}$ is a variety of dimension $\geq 2$ and there are maps $\pi_{n}: X_{n} \rightarrow X_{n-1}$ as above. Then the stack $X_{\infty}$ is a sheaf in the fpqc topology. Further, suppose that the maps $\pi_{n}$ satisfy the following conditions:

(i) For all $n, \pi_{n}^{-1}$ is defined at all but finitely many points of $X_{n-1}^{o}$. That is, the set of exceptional points of $\pi^{-1}: X \rightarrow X_{\infty}$ is countable; let $\left\{z_{m}\right\}_{m \in \mathbb{N}}$ be an enumeration of this set.

(ii) The set $\left\{z_{m}\right\}$ is critically dense.

(iii) For all $m$, there is some $n(m)$ so that, for $n \geq n(m)$, the map $\pi_{n}$ is a local isomorphism at all points in the preimage of $z_{m}$.

(iv) For all $m \in \mathbb{N}$, there is an ideal sheaf $\mathscr{F}_{m}$ on $X$, cosupported at $z_{m}$, so that $X_{n(m)}$ is a closed subscheme of Proj $\mathscr{S}_{y m_{X}} \mathscr{F}_{m}$ above a neighborhood of $z_{m}$. That is, $X_{n(m)} \rightarrow X$ factors as

$$
X_{n(m)} \stackrel{c_{m}}{\longrightarrow} \operatorname{Proj} \mathscr{S y m}_{X} \mathscr{F}_{m} \stackrel{p_{m}}{\longrightarrow} X,
$$

where $p_{m}: \operatorname{Proj} \mathscr{S}_{y m_{X}} \mathscr{F}_{m} \rightarrow X$ is the natural map and $c_{m}$ is a closed immersion over a neighborhood of $z_{m}$.

(v) There is some $D \in \mathbb{N}$ so that $\mathfrak{m}_{z_{m}}^{D} \mathcal{O}_{X, z_{m}} \subseteq \mathscr{g}_{m} \subseteq \mathcal{O}_{X, z_{m}}$ for every $m$.

Then:

(1) The stack $X_{\infty}$ is fpqc-algebraic.

(1a) $X_{\infty}$ has a representable, formally étale, fpqc cover by an affine scheme $U \rightarrow X_{\infty}$

(1b) The diagonal morphism $\Delta: X_{\infty} \rightarrow X_{\infty} \times_{\mathbb{k}} X_{\infty}$ is representable, separated, and quasicompact.

(2) The morphism $\pi: X_{\infty} \rightarrow X$ is quasicompact.

(3) $X_{\infty}$ is noetherian as an fpqc-algebraic stack.

Proof. Because any limit of an inverse system of sheaves taken in the category of presheaves is already a sheaf [Hartshorne 1977, Exercise II.1.12], $X_{\infty}$ is a sheaf in the fpqc topology.

Now assume that (i)-(v) hold. For $n \in \mathbb{N}$, let $W_{n}$ be the scheme-theoretic image of $c_{n}$. Let

$$
X_{n}^{\prime}:=W_{0} \times_{X} W_{1} \times_{X} \cdots \times_{X} W_{n-1} .
$$

Let $\pi_{n}^{\prime}: X_{n}^{\prime} \rightarrow X_{n-1}^{\prime}$ be projection on the first $n-1$ factors. We show we can assume without loss of generality that $X_{n}=X_{n}^{\prime}$; that is, we claim that

$$
\lim _{\pi^{\prime}} X_{n}^{\prime} \cong \lim _{\pi} X_{n} .
$$


Let $k \in \mathbb{N}$. Let $K(k):=\max \{k, n(0), \ldots, n(k-1)\}$. For each $0 \leq m \leq k-1$, there is a morphism

$$
X_{K(k)} \stackrel{\pi^{K(k)-n(m)}}{\longrightarrow} X_{n(m)} \stackrel{c_{m}}{\longrightarrow} W_{m} .
$$

Since these agree on the base, we obtain an induced $\phi_{k}: X_{K(k)} \rightarrow X_{k}^{\prime}$. The $\phi_{k}$ are clearly compatible with the inverse systems $\pi$ and $\pi^{\prime}$. Taking the limit, we obtain

$$
\phi: \lim _{\leftarrow} X_{K(k)} \rightarrow \lim _{\longleftarrow} X_{k}^{\prime} .
$$

Now let $N(k):=k+\max \left\{m \mid z_{m} \in F_{k}\right\}$, where $F_{k}$ is the set of fundamental points of $X \rightarrow X_{k}$. We claim there is a morphism $\psi_{k}: X_{N(k)}^{\prime} \rightarrow X_{k}$. There is certainly a rational map defined over $X \backslash\left\{F_{k}\right\}$ since there $X_{k}$ is locally isomorphic to $X$. Let $z_{m} \in F_{k}$, and let $n^{\prime}(m):=\max \{k, n(m)\}$. The rational map

$$
X_{N(k)}^{\prime} \rightarrow W_{m}-\rightarrow X_{n^{\prime}(m)} \rightarrow X_{k}
$$

is then defined over a neighborhood of $z_{m}$. These maps clearly agree on overlaps, so we may glue to define $\psi_{k}$ as claimed. Let

$$
\psi: \lim _{\leftarrow} X_{N(k)}^{\prime} \rightarrow \lim _{\leftarrow} X_{k}
$$

be the limit of the $\psi_{k}$. It is clear that $\psi=\phi^{-1}$; note that by construction both $N(k)$ and $K(k)$ go to infinity as $k \rightarrow \infty$.

Going forward, we replace $X_{n}$ by $X_{n}^{\prime}$. Thus, let $Y_{n}:=\operatorname{Proj} \mathscr{S}_{y m_{X}} \mathscr{F}_{n}$, and assume that there are closed immersions $i_{n}: X_{n} \rightarrow Y_{0} \times_{X} \cdots \times_{X} Y_{n-1}$ so that the $\pi_{n}$ are given by restricting the projection maps.

It suffices to prove the proposition in the case that $X=\operatorname{Spec} C$ is affine; note that we can choose an affine subset of $X$ that contains all $z_{n}$. Let $J_{n} \subseteq C$ be the ideal cosupported at $z_{n}$ so that $\left(J_{n}\right)_{z_{n}}=\mathscr{J}_{n}$. Let $\mathfrak{m}_{p}$ denote the maximal ideal of $C$ corresponding to $p$.

Claim 4.3. There is an $N$ such that every ideal $J_{m}, m \in \mathbb{N}$, is generated by at most $N$ elements.

Proof. Embed $X$ in an affine space; i.e., choose a closed immersion $X \subseteq \mathbb{A}^{l}$. Then each point of $\mathbb{A}^{l}$, hence a fortiori each point of $X$, is cut out scheme-theoretically by $l$ elements of $C$, and the power of the maximal ideal $\mathfrak{m}_{z_{m}}^{D}$ appearing in hypothesis (v) of the proposition is generated by $N_{0}:=\left(\begin{array}{c}D+l-1 \\ l-1\end{array}\right)$ elements of $C$. Now $J_{m}$ contains $\mathfrak{m}_{z_{m}}^{D}$, and

$$
\operatorname{dim}\left(J_{m} / \mathfrak{m}_{z_{m}}^{D}\right) \leq \operatorname{dim} C / \mathfrak{m}_{z_{m}}^{D} \leq \operatorname{dim} \mathbb{k}\left[u_{1}, \ldots, u_{l}\right] /\left(u_{1}, \ldots, u_{l}\right)^{D}=: N_{1} .
$$

Thus, $J_{m}$ is generated by at most $N:=N_{0}+N_{1}$ elements. 
We continue with the proof of the proposition.

(1a) To construct an affine scheme $U$ with a representable, formally étale morphism $U \rightarrow X_{\infty}$, we proceed as follows. For each $n$ and for each $1 \leq i \leq N$, we choose hypersurfaces $D_{n, i}=V\left(d_{n, i}\right) \subset X$ with the following properties:

(A) For all $n$, the elements $d_{n, 1}, \ldots, d_{n, N}$ generate $J_{n}$.

(B) For all $n$ and $i$, the hypersurface $D_{n, i}$ does not contain any irreducible component $Z$ of a hypersurface $D_{m, j}$ with $m<n$ or $m=n$ and $j<i$.

(C) For all $n$ and each $m \neq n, z_{m} \notin D_{n, i}$ for any $i$.

We can make such choices because $\mathbb{k}$ is uncountable and $X$ is affine (note that in order to satisfy (B), the choice of each $D_{n, i}$ will depend on finitely many earlier choices).

Let $Z_{\left(n_{1}, \ldots, n_{N}\right)}:=D_{n_{1}, 1} \cap \cdots \cap D_{n_{N}, N}$ for each $N$-tuple of positive integers $\left(n_{1}, \ldots, n_{N}\right)$. Note that $z_{m} \notin Z_{\left(n_{1}, \ldots, n_{N}\right)}$ unless $\left(n_{1}, \ldots, n_{N}\right)=(m, m, \ldots, m)$ by property (C). Note also that, since $Z_{\left(n_{1}, \ldots, n_{N}\right)}$ is a union of intersections of pairwise distinct irreducible hypersurfaces, it has codimension at least 2 in $X$.

Now let $Z^{(1)}:=\bigcup_{\left(n_{1}, \ldots, n_{N}\right)} Z_{\left(n_{1}, \ldots, n_{N}\right)}$. This is a countable union of irreducible subsets of $X$ of codimension at least 2 . We may choose one point lying on each component of $Z^{(1)} \backslash\left\{z_{m}\right\}_{m \in \mathbb{N}}$. Now, for each $n$, choose a hypersurface $D_{n, N+1}$ such that $z_{n} \in D_{n, N+1}$, the local ideal of $D_{n, N+1}$ at $z_{n}$ is contained in $\mathscr{g}_{n}$, and $D_{n, N+1}$ avoids all the (countably many) chosen points of components of $Z^{(1)}$ and all $z_{m}$, $m \neq n$. Then for each $n, Z^{(1)} \cap D_{n, N+1}$ is a countable union of irreducible algebraic subsets of codimension at least 3 (it is a union of proper intersections of $D_{n, N+1}$ with irreducible subsets of codimension at least 2). Let $Z^{(2)}:=\bigcup_{n}\left(Z^{(1)} \cap D_{n, N+1}\right)$.

Repeating the previous construction with $Z^{(2)}$, we get hypersurfaces $D_{n, N+2}$ such that each $Z^{(2)} \cap D_{n, N+2}$ is a countable union of irreducible subsets of codimension at least 4 . Iterating, we eventually define hypersurfaces $D_{n, N+i}, i=1, \ldots, d$, with the following properties:

$\left(\mathrm{A}^{\prime}\right)$ For all $m \in \mathbb{N}$, a scheme-theoretic equality $\operatorname{Spec}\left(C / J_{m}\right)=D_{m, 1} \cap \cdots \cap D_{m, N+d}$ exists.

$\left(\mathrm{B}^{\prime}\right)$ For every sequence $\left(n_{1}, \ldots, n_{N+d}\right)$ of positive integers, we have a set-theoretic equality

$$
D_{n_{1}, 1} \cap \cdots \cap D_{n_{N+d}, N+d}= \begin{cases}z_{m} & \text { if }\left(n_{1}, \ldots, n_{N+d}\right)=(m, \ldots, m), \\ \varnothing & \text { otherwise. }\end{cases}
$$

(C) For all $n$ and each $m \neq n, z_{m} \notin D_{n, i}$ for any $i$.

For $0 \leq n \leq m-1$, we abusively let $\tilde{D}_{n, i} \subset X_{m}^{o}$ denote the proper transform of $D_{n, i}$. By construction, $\tilde{D}_{n, 1} \cap \cdots \cap \tilde{D}_{n, N+d}=\varnothing$. 
For each $m \in \mathbb{N}$, the map $C^{N+d} \rightarrow J_{m}, e_{i} \mapsto d_{m, i}$ induces a closed immersion $Y_{m} \rightarrow \mathbb{P}_{X}^{N+d-1}$. Let $V_{m, i} \subseteq Y_{m}$ be the open affine given by $e_{i} \neq 0$. Note that $V_{m, i} \cap X_{m}^{o}=X_{m}^{o} \backslash \tilde{D}_{m, i}$ (recall $X_{m}^{o}$ here denotes the closure in $X_{m}$ of the preimage in $X_{m}$ of the generic point of $X$ ). Let

$$
U_{n, i}:=X_{n} \cap\left(V_{0, i} \times_{X} V_{1, i} \times_{X} \cdots \times_{X} V_{n-1, i} .\right)
$$

The $U_{n, i}$ are open and affine. Since $D_{m, i} \not \supset z_{n}$ for $m \neq n$, the set $\bigcup_{i} U_{n, i}$ includes all irreducible components of $X_{n}$ except possibly for $X_{n}^{o}$. But

$$
X_{n}^{o} \backslash \bigcup_{i=1}^{N+d} U_{n, i}=\bigcap \bigcup_{i}^{n-1} \tilde{D}_{m, i}=\bigcup_{m} \bigcap_{i} \tilde{D}_{m, i}=\varnothing .
$$

Thus, the $U_{n, i}$ are an open affine cover of $X_{n}$.

Since $\left.\pi_{n}\right|_{U_{n, i}}$ is obtained by base extension from the affine morphism $V_{n-1, i} \rightarrow X$, it is affine, and $\pi_{n}\left(U_{n, i}\right) \subseteq U_{n-1, i}$. Writing $C_{i}:=\lim _{\longrightarrow} C_{m, i}$ and $U_{i}:=\operatorname{Spec} C_{i}$, we get $U_{i}=\lim _{\leftarrow} U_{m, i}$; all the $U_{i}$ are affine schemes. By construction, we obtain induced maps $U_{i} \stackrel{\leftarrow}{\rightarrow} X_{\infty}$. Let $U:=\bigsqcup_{i} U_{i}$.

Claim 4.4. The induced morphism $U \rightarrow X_{\infty}$ is representable and formally étale.

Proof. Since each map $U_{i} \rightarrow X_{\infty}$ is a limit of formally étale morphisms, each is itself formally étale. We must show that if $T$ is a scheme equipped with a morphism $T \rightarrow X_{\infty}$, then $T \times_{X_{\infty}} U \rightarrow T$ is a scheme over $T$. Each morphism $T \times_{X_{m}} U_{m, i} \rightarrow T$ is an affine open immersion since the morphisms $U_{m, i} \rightarrow X_{m}$ are affine open immersions. Hence, the morphism $\lim _{\leftarrow}\left(T \times_{X_{m}} U_{m, i}\right) \rightarrow T$ is an inverse limit of schemes affine over $T$ and thus is itself a scheme affine over $T$ [EGA IV.3 1966, Proposition 8.2.3]. The claim now follows from this result:

Lemma 4.5. For any scheme $T$ equipped with a morphism $T \rightarrow X_{\infty}$, we have $T \times_{X_{\infty}} U_{i} \cong \lim _{\leftarrow}\left(T \times_{X_{m}} U_{m, i}\right)$.

Claim 4.6. The map $U \rightarrow X_{\infty}$ is surjective.

Proof. Surjectivity for representable morphisms can be checked locally on the target by [Laumon and Moret-Bailly 2000, 3.10]. Thus, we may change base along a map $T \rightarrow X_{\infty}$ from a scheme $T$, and taking a point that is the image of a map $\operatorname{Spec}(K) \rightarrow T$ where $K$ is a field containing $\mathbb{k}_{\text {, it }}$ suffices to find $\operatorname{Spec}(K) \rightarrow U$ making the diagram

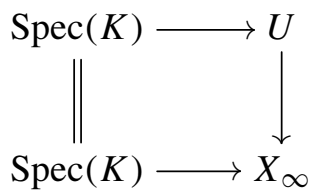

commute. 
Thus, suppose we are given a map $\operatorname{Spec}(K) \rightarrow X_{\infty}$; let $y_{n}$ denote its image (i.e., the image of the unique point of $\operatorname{Spec}(K))$ in $X_{n}$. Let $I_{n}$ denote the (finite) set of those $i$ so that $y_{n} \in U_{n, i}$. Since the $U_{n, i}$ cover $X_{n}$, each $I_{n}$ is nonempty; further, $I_{n} \subseteq I_{n-1}$. The intersection $\bigcap_{n} I_{n}$ is thus nonempty and contains some $i_{0}$. The maps $\operatorname{Spec}(K) \rightarrow U_{m, i_{0}}$ for $m \gg 0$ define a map $f: \operatorname{Spec}(K) \rightarrow U_{i_{0}}=\lim _{\longleftarrow} U_{m, i_{0}} \subset U$, and thus, defining the map in the top row of (4.7) to be $f$ gives the desired commutative diagram. This proves the claim.

Returning to the proof of Proposition 4.2(1a), let $R:=U \times_{X_{\infty}} U$. If we define $R_{i j}:=U_{i} \times_{X_{\infty}} U_{j}$, then we have $R=\bigsqcup_{i, j} R_{i j}$. Note that $R_{i j}$ is a scheme affine over $U_{i}$ by the previous paragraph. Since affine schemes are quasicompact, this proves that the morphism $U \rightarrow X_{\infty}$ is quasicompact. Furthermore, $O\left(R_{i j}\right)$ is a localization of $C_{i}$ (obtained by inverting the images of the elements $d_{n, j}$ ), so $R_{i j} \rightarrow U_{i}$ is flat. We have already proved that $U \rightarrow X_{\infty}$ is surjective, so we conclude that $U \rightarrow X_{\infty}$ is faithfully flat. It follows that $U \rightarrow X_{\infty}$ is fpqc using [Vistoli 2005, Proposition 2.33(iii)]. This completes the proof of (1a).

(1b) The diagonal $\Delta: X_{\infty} \rightarrow X_{\infty} \times_{\mathbb{k}} X_{\infty}$ is the inverse limit of the diagonals $\Delta_{n}: X_{n} \rightarrow X_{n} \times_{\mathbb{k}} X_{n}$. Similarly to Lemma 4.5 , if $V \rightarrow X_{\infty} \times_{\mathbb{k}} X_{\infty}$ is any morphism from a scheme $V$, we get $X_{\infty} \times_{X_{\infty} \times_{\mathbb{k}} X_{\infty}} V \cong \lim X_{n} \times_{X_{n} \times_{\mathbb{k}} X_{n}} V$. Since each $X_{n}$ is separated over $\mathbb{k}$, each morphism $X_{n} \times_{X_{n} \times{ }_{k} X_{n}} V \rightarrow V$ is a closed immersion; hence, $X_{\infty} \times_{X_{\infty} \times_{\mathbb{k}}} X_{\infty} V \rightarrow V$ is a closed immersion. This proves (1b).

(2) Again, we may assume that $X$ is affine. Then, as above, we have an fpqc cover $U \stackrel{p}{\rightarrow} X_{\infty}$ by an affine scheme $U$. Since an affine scheme is quasicompact and a continuous image of a quasicompact space is quasicompact, $X_{\infty}$ is quasicompact as desired.

(3) By our definition, it suffices to prove that $U$ is noetherian or, equivalently, that each $C_{i}$ is a noetherian ring. This follows as in [Artin et al. 1999, Theorem 1.5]. We will need the following:

Lemma 4.8. Let $A$ be a commutative noetherian ring, and (with $M$ an $n \times m$ matrix acting by left multiplication) let $J$ be an ideal of $A$ with a resolution

$$
A^{m} \stackrel{M}{\rightarrow} A^{n} \rightarrow J \rightarrow 0 .
$$

Let $A^{\prime}:=A\left[t_{1}, \ldots, t_{n-1}\right] /\left(t_{1}, \ldots, t_{n-1}, 1\right) M$. Let $P^{\prime}$ be a prime of $A^{\prime}$, and let $P:=P^{\prime} \cap A$. If $P$ and $J$ are comaximal, then $P A^{\prime}=P^{\prime}$.

Note that $A^{\prime}$ is the coordinate ring of a chart of $\operatorname{Proj}_{\operatorname{Sym}_{A}} J$.

Proof. We may localize at $P$, so without loss of generality, $J=A$. Then $A^{\prime} \cong A\left[g^{-1}\right]$ for some $g \in A$. The result follows.

We return to the proof of (3). It suffices to show, by [Eisenbud 1995, Exercise 
2.22], that each prime of $C_{i}$ is finitely generated. Let $\tilde{P} \neq 0$ be a prime of $C_{i}$. Let $P:=\tilde{P} \cap C$, and let $P_{n}:=\tilde{P} \cap C_{n, i}$. By critical density, there is some $n \in \mathbb{N}$ so that if $m \geq n$, then $J_{m}$ and $P$ are comaximal. It follows from Lemma 4.8 that $P_{m}=P_{n} C_{m, i}$ for $m \geq n$. So $\tilde{P}=\bigcup P_{m}=P_{n} C_{i}$. This is finitely generated because $C_{n, i}$ is noetherian, so $P_{n}$ is finitely generated.

Proposition 4.2 is now proved.

Corollary 4.9. Let $X$ be a projective variety, let $\sigma \in \operatorname{Aut}_{k}(X)$, and let $\mathscr{L}$ be a $\sigma$-ample invertible sheaf on $X$. Let $P$ be a zero-dimensional subscheme of $X$, all of whose points have critically dense $\sigma$-orbits. Let $\Phi_{n}:=\Phi_{P} \Phi_{P}^{\sigma} \ldots \Phi_{P}^{\sigma^{n-1}}$. Let $a_{n}: X_{n} \rightarrow X$ be the blowup of $X$ at $\Phi_{n}$ as in Corollary 3.3. Let $\alpha_{n}: X_{n} \rightarrow X_{n-1}$ be given by Corollary 3.3. Then the limit

$$
X_{\infty}:=\lim _{\longleftarrow} X_{n}
$$

is a noetherian fpqc-algebraic stack.

Proof. This follows immediately from Proposition 4.2.

\section{Moduli schemes for truncated point modules}

Let $X$ be a projective variety, let $\sigma \in \operatorname{Aut}_{k}(X)$, and let $\mathscr{L}$ be a $\sigma$-ample invertible sheaf on $X$. Let $P$ be a zero-dimensional subscheme of $X$, all of whose points have critically dense $\sigma$-orbits. We define $\mathscr{Y}:=\mathscr{S}(X, \mathscr{L}, \sigma, P)$ and $S:=S(X, \mathscr{L}, \sigma, P)$ as in Section 2. As usual, we assume that $S$ is generated in degree 1.

In this section, we construct moduli schemes of truncated point modules over $\mathscr{S}$ and $S$. In the next section, we compare them. We begin by constructing moduli schemes for shifted point modules for an arbitrary connected graded noetherian algebra generated in degree 1, generalizing slightly results of [Artin et al. 1990; Rogalski and Stafford 2009].

Let $C$ be any commutative $\mathbb{k}$-algebra. Recall that we use subscript notation to

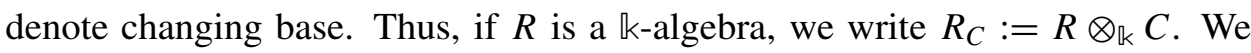
write $X_{C}:=X \times_{\mathbb{k}}$ Spec $C$. Recall that a $C$-point module (over $R$ ) is a graded factor $M$ of $R_{C}$ so that $M_{i}$ is rank-1 projective for $i \geq 0$. An $l$-shifted $C$-point module (over $R$ ) is a factor of $\left(R_{C}\right)_{\geq l}$ that is rank-1 projective in degree $\geq l$. A truncated $l$-shifted $C$-point module of length $m$ is a factor module of $\left(R_{C}\right)_{\geq l}$ so that $M_{i}$ is rank-1 projective over $C$ for $l \leq i \leq l+m-1$ and $M_{i}=0$ for $i \geq l+m$. Since these modules depend on a finite number of parameters, they are clearly parametrized up to isomorphism by a projective scheme. For fixed $l \leq n$, we let $Y_{n}$ denote the $l$-shifted length- $(n-l+1)$ point scheme of $R$. A point in ${ }_{l} Y_{n}$ gives a surjection $R_{\geq l} \rightarrow M$ (up to isomorphism) or equivalently a submodule of $R_{\geq l}$ with appropriate Hilbert series. Thus, we say that ${ }_{l} Y_{n}$ parametrizes embedded (shifted truncated) point modules. The map $M \mapsto M / M_{n}$ induces a morphism $\chi_{n}:{ }_{l} Y_{n} \rightarrow{ }_{l} Y_{n-1}$. 
For later use, we explicitly construct a projective embedding of ${ }_{l} Y_{n}$.

Proposition 5.1 [Artin et al. 1990, Section 3]. Let $R$ be a connected graded $\mathbb{k}$ algebra generated in degree 1.

(1) For all $l \leq n \in \mathbb{N}$, there is a closed immersion

$$
{ }_{l} \Pi_{n}:{ }_{l} Y_{n} \rightarrow \mathbb{P}\left(\left(R_{1}^{\otimes l}\right)^{\vee}\right) \times \mathbb{P}\left(R_{1}^{\vee}\right)^{\times(n-l)} .
$$

(2) Fix $l \leq n$, and let

$$
\pi: \mathbb{P}\left(\left(R_{1}^{\otimes l}\right)^{\vee}\right) \times \mathbb{P}\left(R_{1}^{\vee}\right)^{\times(n-l)} \rightarrow \mathbb{P}\left(\left(R_{1}^{\otimes l}\right)^{\vee}\right) \times \mathbb{P}\left(R_{1}^{\vee}\right)^{\times(n-l-1)}
$$

be projection onto the first $n-l$ factors. Then the following diagram commutes:

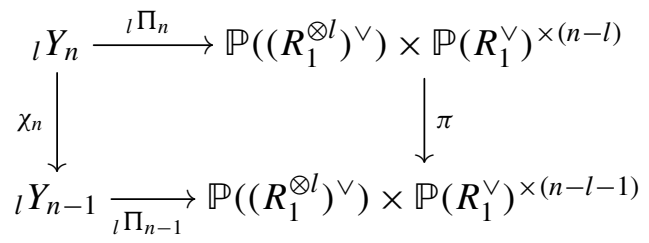

Proof. (1) Let $T=T^{\bullet}\left(R_{1}\right)$ denote the tensor algebra on the finite-dimensional k-vector space $R_{1}$. We identify $T_{1}$ canonically with $R_{1}$ and $T_{l}$ with $R_{1}^{\otimes l}$.

Given an element $f \in T_{n}$, we get an $(l, 1, \ldots, 1)$-form

$$
\tilde{f}: T_{l}^{\vee} \times\left(T_{1}^{\vee}\right)^{\times(n-l)} \rightarrow \mathbb{k}
$$

by pairing with $f$. The map is $\mathbb{k}$-multilinear; hence, $\tilde{f}$ defines a hypersurface $Y(\tilde{f})$ in $\mathbb{P}\left(T_{l}^{\vee}\right) \times\left(\mathbb{P}\left(T_{1}^{\vee}\right)\right)^{\times(n-l)}$. More generally, given a collection $\left\{f_{i}\right\}$ of elements of $T_{n}$, we get a closed subscheme

$$
Y\left(\left\{\tilde{f}_{i}\right\}\right) \subseteq \mathbb{P}\left(T_{l}^{\vee}\right) \times\left(\mathbb{P}\left(T_{1}^{\vee}\right)\right)^{\times(n-l)} .
$$

Let $I$ be the kernel of the natural surjection $T \rightarrow R$. Then the above construction gives a closed subscheme $Y\left(\tilde{I}_{n}\right) \subseteq \mathbb{P}\left(T_{l}^{\vee}\right) \times\left(\mathbb{P}\left(T_{1}^{\vee}\right)\right)^{\times(n-l)}$. We claim that $Y\left(\tilde{I}_{n}\right)$ is naturally isomorphic to ${ }_{l} Y_{n}$.

Let $C$ be a commutative $\mathbb{k}$-algebra, and let $R_{C}=R \otimes_{\mathbb{k}} C$ and $T_{C}=T \otimes_{\mathbb{k}} C$ with the gradings induced from $R$ and $T$, respectively. Suppose that $\bar{\alpha}:\left(R_{C}\right)_{\geq l} \rightarrow M$ is an embedded $l$-shifted truncated $C$-point module of length $n-l+1$. We write $\alpha$ : $\left(T_{C}\right)_{\geq l} \rightarrow M$ for the composite of the two surjections. Assume that $M=\bigoplus_{i=l}^{n} m_{i} \cdot C$ is a free graded $C$-module on generators $m_{i}$. Then $\alpha$ determines $C$-linear maps $a_{j}: T_{1} \otimes_{\mathbb{k}} C \rightarrow C$ for $1 \leq j \leq n-l$ by $m_{l+j-1} x=m_{l+j} a_{j}(x)$ for $x \in T_{1} \otimes_{\mathbb{k}} C$ and a $C$-linear map $b: T_{l} \otimes_{\mathbb{k}} C \rightarrow C$ by $\alpha(y)=m_{l} b(y)$ for $y \in T_{l} \otimes_{\mathbb{k}} C$. Since $M$ is a (shifted truncated) point module, hence generated in degree $l$, these maps are 
surjective. Hence, they determine a morphism

$$
\Pi(\alpha)=\left(b, a_{1}, \ldots, a_{n-l}\right): \operatorname{Spec}(C) \rightarrow \mathbb{P}\left(T_{l}^{\vee}\right) \times\left(\mathbb{P}\left(T_{1}^{\vee}\right)\right)^{\times(n-l)} .
$$

We see immediately from the construction that if $f \in I_{n} \otimes_{\mathbb{k}} C$, then $\tilde{f}(\Pi(\alpha))=0$. In particular, $\Pi(\alpha)$ factors through $Y\left(\tilde{I}_{n}\right)$.

It follows immediately that the above construction defines a morphism $\Pi$ from the moduli functor of shifted truncated point modules with free (as $C$-modules) graded components to $Y\left(\tilde{I}_{n}\right)$. Since the latter is a scheme, hence a sheaf in the fpqc topology, $\Pi$ induces a morphism, which we denote ${ }_{l} \Pi_{n}$, from the moduli functor ${ }_{l} Y_{n}$ for all shifted truncated $C$-point modules over $R$ to $Y\left(\tilde{I}_{n}\right)$.

Claim 5.3. The morphism ${ }_{l} \Pi_{n}$ is an isomorphism; that is, $Y\left(\tilde{I}_{n}\right) \cong{ }_{l} Y_{n}$ represents the moduli functor of embedded truncated $l$-shifted $C$-point modules over $R$ of length $n-l+1$.

Proof. A morphism $\operatorname{Spec}(C) \rightarrow Y\left(\tilde{I}_{n}\right) \subseteq \mathbb{P}\left(T_{l}^{\vee}\right) \times\left(\mathbb{P}\left(T_{1}^{\vee}\right)\right)^{\times(n-l)}$ gives a tuple $\left(b, a_{1}, \ldots, a_{n-l}\right)$ where each $a_{j}$ is a surjective $C$-linear map $a_{j}: T_{1} \otimes_{\mathbb{k}} C \rightarrow N_{j}$ and each $N_{j}$ is a finitely generated projective $C$-module of rank 1 ; and $b: T_{l} \otimes_{\mathbb{k}} C \rightarrow M_{l}$ is a surjective $C$-linear map onto a finitely generated projective $C$-module $M_{l}$ of rank 1.

Assume first that $M_{l}$ and each $N_{j}$ is a free $C$-module, and choose basis elements. Define a $T_{C}$-module $M=\bigoplus_{j=l}^{n} m_{j} \cdot C$ by $m_{j-1} x=m_{j} a_{j-l}(x)$ for $x \in T_{1} \otimes_{\mathbb{k}} C$. Moreover, define a map $\alpha:\left(T_{C}\right)_{\geq l} \rightarrow M$ by $\alpha(y)=m_{l} b(y)$ for $y \in T_{l}$ and extending linearly. It is a consequence of the construction of $Y\left(\tilde{I}_{n}\right)$ that the map $\alpha$ factors through $\left(R_{C}\right)_{\geq l}$ and makes $M$ an $l$-shifted truncated $C$-point module over $R$.

Next, we observe that the functor $\Pi$ (on shifted truncated point modules with free $C$-module components) and the above construction (on maps $\operatorname{Spec}(C) \rightarrow Y\left(\tilde{I}_{n}\right)$ for which the modules $N_{j}$ and $M_{l}$ are free $C$-modules) give mutual inverses. This follows from the argument of [Artin et al. 1990, 3.9], which uses only the freeness condition. In particular, the functor $\Pi$ is injective.

To prove that the sheafification ${ }_{l} \Pi_{n}$ is an isomorphism, then it suffices to show that $\Pi$ is locally surjective; that is, for every morphism $\operatorname{Spec}(C) \rightarrow Y\left(\tilde{I}_{n}\right)$, there is a faithfully flat morphism $\operatorname{Spec}\left(C^{\prime}\right) \rightarrow \operatorname{Spec}(C)$ and a shifted truncated $C^{\prime}$-point module with free $C^{\prime}$-module components, whose image under $\Pi$ is the composite map $\operatorname{Spec}\left(C^{\prime}\right) \rightarrow Y\left(\tilde{I}_{n}\right)$. But it is standard that such a homomorphism $C \rightarrow C^{\prime}$ can be found that makes each $N_{j}$ and $M_{l}$ trivial, and now the construction of the previous paragraph proves the existence of the desired shifted truncated point module. This completes the proof of the claim.

Now part (1) of the proposition follows from the claim, and (2) follows by construction. 
Proposition 5.4 [Artin et al. 1990, Proposition 3.6]. Let $R$ be a connected graded $\mathbb{k}$-algebra generated in degree 1 . Let $n>l$, and consider the truncation morphism

$$
\chi_{n}:{ }_{l} Y_{n} \rightarrow{ }_{l} Y_{n-1} .
$$

Let $y \in_{l} Y_{n-1}$, and suppose that $\operatorname{dim} \chi_{n}^{-1}(y)=0$. Then $\chi_{n}^{-1}$ is defined and is a local isomorphism locally in a neighborhood of $y$.

Proof. We consider the commutative diagram (5.2) of Proposition 5.1(2). By Proposition 5.1(1), the horizontal maps are closed immersions. Since the defining equations of $Y\left(\tilde{I}_{n}\right) \subseteq \mathbb{P}\left(T_{l}^{\vee}\right) \times\left(\mathbb{P}\left(T_{1}^{\vee}\right)\right)^{\times(n-l)}$ are $(l, 1, \ldots, 1)$-forms and in particular are linear in the last coordinate, the fibers of $\chi_{n}$ are linear subspaces of $\mathbb{P}\left(T_{1}^{\vee}\right)$. The result follows as in the proof of [Artin et al. 1990, Proposition 3.6(ii)].

Proposition 5.5 [Rogalski and Stafford 2009, Proposition 2.5]. Let $R$ be a noetherian connected graded $\mathbb{k}$-algebra generated in degree 1 . For $n>l \geq 0$, define $\chi_{n}:{ }_{l} Y_{n} \rightarrow{ }_{l} Y_{n-1}$ as in the beginning of the section. Let $n_{0} \geq 0$, and let $\left\{y_{n} \in{ }_{l} Y_{n} \mid n \geq n_{0}\right\}$ be a sequence of (not necessarily closed) points so that $\chi_{n}\left(y_{n}\right)=y_{n-1}$ for all $n>n_{0}$. Then for all $n \gg n_{0}$, the fiber $\chi_{n}^{-1}\left(y_{n-1}\right)$ is a singleton and $\chi_{n}^{-1}$ is defined and is a local isomorphism at $y_{n-1}$.

Proof. This follows as in the proof of [Rogalski and Stafford 2009, Proposition 2.5], using Proposition 5.4 instead of [Artin et al. 1990, Proposition 3.6(ii)].

We are interested in studying the $\operatorname{limit}_{l} Y_{\infty}:=\lim _{l} Y_{n}$; however, we first study the point schemes of $\mathscr{Y}$. That is, for $n \geq l \geq 0$, it is clear that we may also define a scheme that parametrizes factor modules $\mathcal{M}$ of $\mathscr{S}_{\geq l}$ so that, as graded $\mathrm{O}_{X}$-modules, $M \cong \mathbb{k}_{x} t^{l} \oplus \cdots \oplus \mathbb{k}_{x} t^{n}$ for some $x \in X$. We say that $x$ is the support of $\mathcal{M}$. We let ${ }_{l} Z_{n}$ denote this $l$-shifted length- $(n-l+1)$ truncated point scheme of $\mathscr{Y}$. More formally, a $\operatorname{Spec}(C)$-point of $Z_{l}$ will be a factor module $\mathcal{M}$ of $\mathscr{Y}_{\geq l} \otimes_{\mathbb{k}} C$ that is isomorphic as a graded $\mathrm{O}_{X_{C}}$-module to a direct sum $P_{l} \oplus \cdots \oplus P_{n}$, where each $P_{i}$ is a coherent ${ }^{O} X_{C}$-module that is finite over $C$ (in the sense that its support in $X_{C}$ is finite over $\operatorname{Spec}(C)$ ) and is a rank-1 projective $C$-module (which is well defined because of the finite support condition). We let $Z_{n}:={ }_{0} Z_{n}$ be the unshifted length- $(n+1)$ point scheme of $\mathscr{Y}$.

For all $n>l \geq 0$, there are maps

$$
\phi_{n}:{ }_{l} Z_{n} \rightarrow{ }_{l} Z_{n-1} \quad \text { defined by } \quad \mu \mapsto M / M_{n} .
$$

If $l=0$ and $\mathcal{M}$ is a truncated point module of length $n$ over $\mathscr{S}$, then $\mathcal{M}[1]_{\geq 0}$ is also cyclic (since $\mathscr{Y}$ is generated in degree 1 ) and so is a factor of $\mathscr{Y}$ in a unique way up to a scalar. This induces a map

$$
\psi_{n}: Z_{n} \rightarrow Z_{n-1} \quad \text { defined by } \quad M \mapsto M[1]_{\geq 0} .
$$

It is clear that $\psi_{n}$ and $\phi_{n}$ map relevant components to relevant components. 
Lemma 5.6. Let $f_{n}:{ }_{l} Z_{n} \rightarrow X$ be the map that sends a module $\mathcal{M}$ to its support. The following diagrams commute:
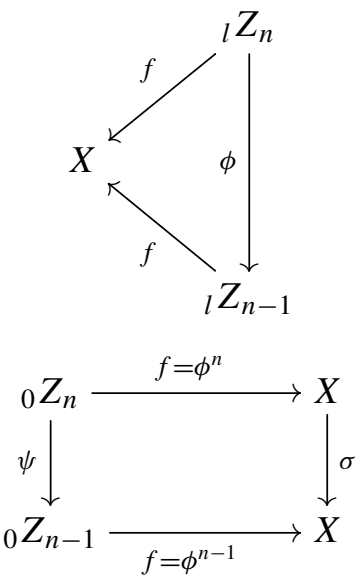

Proof. It is clear by construction that if $\mathcal{M}$ is a shifted truncated point module and $\mathcal{M}^{\prime}$ is a further factor of $\mathcal{M}$, then $\mathcal{M}$ and $\mathcal{M}^{\prime}$ have the same support. Thus, (5.7) commutes.

Let $M$ be a truncated point module corresponding to a point $z \in{ }_{0} Z_{n}$. Let $x:=f(z)$. By [Keeler et al. 2005, Lemma 5.5], we have $M[1]_{n} \cong\left(M_{n+1}\right)^{\sigma^{-1}} \cong\left(\mathbb{k}_{x}\right)^{\sigma^{-1}} \cong \mathbb{k}_{\sigma(x)}$. Thus, $f \psi(z)=\sigma f(z)$ as claimed, and (5.8) commutes.

Recall that $\mathscr{Y}_{n}=\Phi_{n} \mathscr{L}_{n}$.

Proposition 5.9. For $n \geq 0$, let $X_{n}$ be the blowup of $X$ at $\Phi_{n}$. Let $\alpha_{n}, \beta_{n}: X_{n} \rightarrow X_{n-1}$ be as in Corollary 3.3.

Then for all $n>l \geq 0$, there are isomorphisms $j_{n}: X_{n} \rightarrow{ }_{l} Z_{n}^{o} \subseteq{ }_{l} Z_{n}$ so that the following diagrams commute:
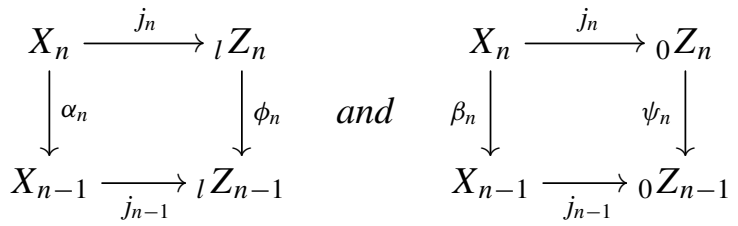

Proof. We will do the case that $l=0$; the general case is similar. Let $Z_{n}:={ }_{0} Z_{n}$. For $0 \leq i \leq n$, let $W_{i}=\operatorname{Proj} \mathscr{S}_{y m_{X}} \mathscr{I}_{i}$ be the scheme parametrizing colength-1 ideals inside $\Phi_{i}$, and let $c_{i}: X_{i} \rightarrow W_{i}$ be the map from Lemma 3.1. Let

$$
r_{n}: X_{n} \rightarrow X \times W_{1} \times \cdots \times W_{n}
$$

be the composition

$$
X_{n} \stackrel{\alpha^{n} \times \alpha^{n-1} \times \cdots \times 1}{\longrightarrow} X \times X_{1} \times \cdots \times X_{n} \stackrel{c_{0} \times \cdots \times c_{n}}{\longrightarrow} X \times W_{1} \times \cdots \times W_{n} .
$$


Since this is the composition of the graph of a morphism with a closed immersion, it is also a closed immersion and is an isomorphism onto its image.

Now, a point in $Z_{n}$ corresponds to an ideal $\mathscr{I} \subset \mathscr{Y}$ so that the factor is a truncated point module of length $n+1$, and there is thus a canonical closed immersion $\delta_{n}: Z_{n} \rightarrow X \times W_{1} \times \cdots \times W_{n}$. The map $\delta_{n}$ sends a graded right ideal $\mathscr{F}$ of $\mathscr{S}$ to the tuple $\left(\mathscr{E}_{0}, \mathscr{E}_{1}, \ldots, \mathscr{E}_{n}\right)$.

Conversely, a point $\left(\mathscr{E}_{0}, \ldots, \mathscr{E}_{n}\right) \in X \times W_{1} \times \cdots \times W_{n}$ is in $\operatorname{Im}(\delta)$ if and only if we have $\mathscr{F}_{i} \mathscr{Y}_{j}^{\sigma^{i}} \subseteq \mathscr{F}_{i+j}$ for all $i+j \leq n$. It follows from Lemma 3.2(c) that $\operatorname{Im}\left(r_{n}\right) \subseteq \operatorname{Im}\left(\delta_{n}\right)$. Since $r_{n}$ and $\delta_{n}$ are closed immersions and $X_{n}$ is reduced, we may define $j_{n}=\delta_{n}^{-1} r_{n}: X_{n} \rightarrow Z_{n}$.

Let $U:=X \backslash \operatorname{Cosupp} \Phi_{n}$. Then $f_{n}^{-1}$ and $a_{n}^{-1}$ are defined on $U$, and the diagram

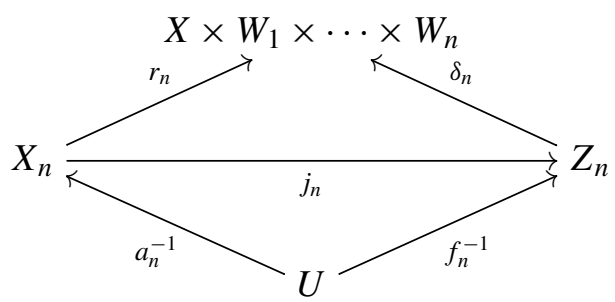

commutes. Since $r_{n}$ and $\delta_{n}$ are closed,

$$
r_{n}\left(X_{n}\right)=r_{n}\left(\overline{a_{n}^{-1}(U)}\right)=\overline{r_{n} a_{n}^{-1}(U)}=\overline{\delta_{n} f_{n}^{-1}(U)}=\delta_{n}\left(\overline{f_{n}^{-1}(U)}\right)=\delta_{n}\left(Z_{n}^{o}\right) .
$$

Therefore, $j_{n}$ is an isomorphism to $Z_{n}^{o}$.

Let $q: X \times W_{1} \times \cdots \times W_{n} \rightarrow X \times W_{1} \times \cdots \times W_{n-1}$ be projection onto the first $n$ factors. Consider the diagram

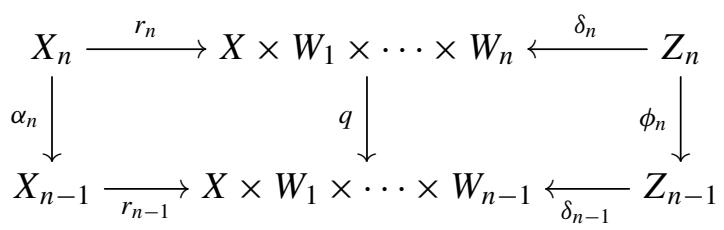

From the definitions of $r_{n}$ and $\delta_{n}$, we see that this diagram commutes; since $j_{n}=\delta_{n}^{-1} r_{n}$, the following diagram commutes:

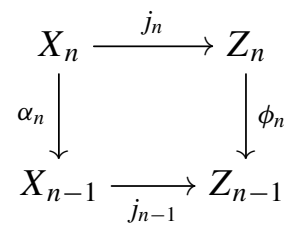

Let $X_{n}^{\prime}:=\operatorname{Im}\left(r_{n}\right) \subset X \times W_{1} \times \cdots \times W_{n}$, and let $\beta_{n}^{\prime}: X_{n}^{\prime} \rightarrow X_{n-1}^{\prime}$ be the map induced from $\beta_{n}$. The proof of Corollary 3.3 shows that if $\left(\mathscr{E}_{0}, \mathscr{F}_{1}, \ldots, \mathscr{F}_{n}\right) \in X_{n}^{\prime}$, 
then its image under $\beta_{n}^{\prime}$ is

$$
\beta_{n}^{\prime}\left(\left(\mathscr{F}_{0}, \mathscr{F}_{1}, \ldots, \mathscr{\Phi}_{n}\right)\right)=\left(\mathscr{F}_{0}, \ldots, \mathscr{F}_{n-1}\right) \in X_{n-1}^{\prime},
$$

where $\mathscr{F}_{i}:=\left(\mathscr{F}_{i+1}: \mathscr{I}_{1}\right)^{\sigma^{-1}} \cap \mathscr{I}_{i}$.

Now let $\mathscr{F}$ be the ideal defining a truncated point module of length $n+1$. By abuse of notation, we think of $\mathscr{E}$ as a point in $Z_{n}$. Let $\mathcal{M}:=\mathscr{S} / \mathscr{\mathscr { S }}$. Then we have $\psi(\mathscr{F})_{i}=\left(\operatorname{Ann}_{\varphi}\left(\mathcal{M}_{1}\right)\right)_{i}$ for $0 \leq i \leq n-1$. Thus, $\mathscr{I}_{1}\left(\psi(\mathscr{F})_{i}\right)^{\sigma} \subseteq \mathscr{I}_{i+1}$ or

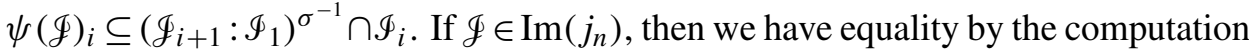
in (5.10). Thus, the diagram

commutes.

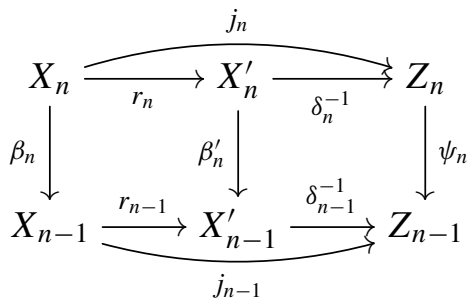

To end this section, we construct stacks ${ }_{l} Z_{\infty}$ and ${ }_{l} Y_{\infty}$ that are fine moduli spaces for (shifted) embedded point modules and give some of their properties. A version of the following result was known long ago to M. Artin; however, it does not seem to have appeared in the literature:

Theorem 5.11. Fix $l \in \mathbb{N}$. For $n \geq l$, let $X_{n}$ be the blowup of $X$ at $\Phi_{n}$. Let ${ }_{l} Y_{n}$ be the moduli space of $l$-shifted length- $(n-l+1)$ point modules over $S$. Let ${ }_{l} Z_{n}$ be the moduli space of $l$-shifted length- $(n-l+1)$ point modules over $Y$. Define the morphisms $\chi_{n}:{ }_{l} Y_{n} \rightarrow{ }_{l} Y_{n-1}, \phi_{n}:{ }_{l} Z_{n} \rightarrow{ }_{l} Z_{n-1}$, and $\alpha_{n}: X_{n} \rightarrow X_{n-1}$ as above. Let

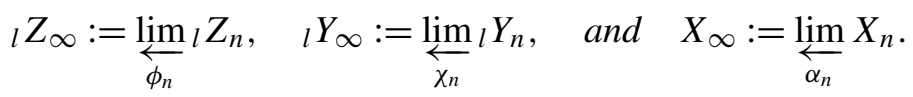

Then the stack ${ }_{l} Y_{\infty}$ is a sheaf in the fpqc topology and is a fine moduli space for $l$ shifted embedded point modules over $S$. The stack $Z_{\infty}$ is noetherian fpqc-algebraic and is a fine moduli space for $l$-shifted embedded point modules over $\mathscr{Y}$. The relevant component of $Z_{\infty} Z_{\infty}$ is isomorphic to $X_{\infty}$.

Proof. We suppress the subscript $l$ in the proof.

For $n \geq l$, let $F_{n}$ be the moduli functor for truncated $l$-shifted point modules over $S$, so $Y_{n} \cong F_{n}$. Define a contravariant functor

$$
F: \text { Affine schemes } \rightarrow \text { Sets, }
$$

Spec $C \mapsto\{$ Embedded $l$-shifted $C$-point modules over $S\}$.

By descent theory, $F$ is a sheaf in the fpqc topology. More precisely, recall that quasicoherent sheaves form a stack in the fpqc topology [Vistoli 2005, Section 4.2.2]; 
consequently, the (graded) quotients of $S_{\geq l}$ form a sheaf of sets in the fpqc topology. Moreover, as in the first paragraph of [Vistoli 2005, Section 4.2.3], those quotients of $S_{\geq l}$ that are $S$-module quotients form a subsheaf in the fpqc topology; this subsheaf is $F$. It is formal that $F$ is isomorphic to the functor $h_{Y_{\infty}}$.

Likewise, $Z_{\infty}$ parametrizes $l$-shifted point modules over $\mathscr{Y}$. We show that (i)-(v) of Proposition 4.2 apply to $Z_{\infty}$. We have $\mathscr{Y}_{n}=\mathscr{I}_{n} \mathscr{L}_{n}$; let $P_{n} \subset X$ be the subscheme defined by $\rrbracket_{n}$. Consider the maps

$$
Z_{n} \stackrel{\phi_{n}}{\longrightarrow} Z_{n-1} \stackrel{f_{n-1}}{\longrightarrow} X
$$

from Lemma 5.6. Now, $f_{n}^{-1}$ is defined away from $P_{n}$, and $\bigcup_{n} P_{n}$ is a countable critically dense set. Thus, (i) and (ii) hold.

Let $x \in \bigcup P_{n}$. As the points in $P$ have infinite orbits, there is some $m \in \mathbb{N}$ so that $x \notin \sigma^{-n}(P)$ for all $n \geq m$. Let $z_{m} \in Z_{m}$ with $f_{m}\left(z_{m}\right)=x$, corresponding to a right ideal $\mathscr{\mathscr { S }} \subseteq \mathscr{Y}_{\geq l}$ with $S_{\geq l} / \mathscr{\mathscr { S }} \cong \bigoplus_{j=l}^{m} \mathcal{O}_{x}$. Let $\mathscr{J}^{\prime}:=\mathscr{S}_{\leq m} \cdot \mathscr{Y}$. For any $j \geq 0$, we have $\left(\mathscr{S}_{j}^{\sigma^{m}}\right)_{x}=\left(\mathscr{L}_{j}^{\sigma^{m}}\right)_{x}$, and so $\mathscr{F}^{\prime}$ gives the unique preimage of $z_{m}$ in $Z_{\infty}$. A similar uniqueness holds upon base extension, so the scheme-theoretic preimage of $z_{m}$ in $Z_{\infty}$ is a $\mathbb{k}$-point, and $\Phi_{m}^{-1}$ is defined and is a local isomorphism at $z_{m}$.

For $j \geq l$, let $W_{j}:=\operatorname{Proj} \mathscr{S}_{y m_{X}} \Phi_{n}$. As in the proof of Proposition 5.9, we may regard $Z_{n}$ as a closed subscheme of $W_{l} \times \cdots \times W_{n}$, and (iv) and (v) follow from this and the fact that the orbits of points in $P$ are infinite. By Proposition 4.2, then $Z_{\infty}$ is a noetherian fpqc-algebraic stack.

Consider the morphisms $j_{n}: X_{n} \stackrel{\cong}{\rightrightarrows} Z_{n}^{o} \subseteq Z_{n}$ from Proposition 5.9. Commutativity of the first diagram there gives an induced isomorphism $j: X_{\infty} \rightarrow Z_{\infty}^{o} \subseteq Z_{\infty}$.

\section{Comparing moduli of points}

In this section, we prove that ${ }_{l} Y_{\infty}$ is also noetherian fpqc-algebraic and that, at least for sufficiently large $l$, the stacks ${ }_{l} Z_{\infty}$ and ${ }_{l} Y_{\infty}$ are isomorphic.

In the following pages, we will always use the following notation. We write a commutative $\mathbb{k}$-algebra $C$ as $p: \mathbb{k} \rightarrow C$ to indicate the structure map explicitly. We write $X_{C}:=X \otimes_{\mathbb{k}}$ Spec $C$. We abuse notation and let $p$ also denote the projection map $1 \otimes p: X_{C} \rightarrow X$. We let $q: X_{C} \rightarrow$ Spec $C$ be projection on the second factor.

Suppose that $p: \mathbb{k} \rightarrow C$ is a commutative $\mathbb{k}$-algebra and $y: \operatorname{Spec} C \rightarrow X$ is a $C$-point of $X$. Then $y$ determines a section of $q$, which we also call $y$. This is a morphism $y:$ Spec $C \rightarrow X_{C}$. We define $\Phi_{y} \subseteq O_{X_{C}}$ to be the ideal sheaf of the corresponding closed subscheme of $X_{C}$. We define $\mathcal{O}_{y}:=\mathfrak{O}_{X_{C}} / \mathscr{I}_{y}$.

We use the relative regularity results from Section 2 to study the pullbacks of the sheaves $\mathscr{S}_{n}$ to $X_{C}$. Fix a very ample invertible sheaf $\mathcal{O}_{X}(1)$ on $X$, which we will use to measure regularity. 
Lemma 6.1. Suppose $p: \mathbb{k} \rightarrow C$ is a commutative noetherian $\mathbb{k}$-algebra. Then $\left\{p^{*} \mathscr{S}_{n}\right\}_{n \geq 0}$ is a right ample sequence on $X_{C}$.

Proof. Let $\mathscr{F}$ be a coherent sheaf on $X_{C}$. By [Rogalski and Stafford 2007, Corollary 3.14], $\lim _{n \rightarrow \infty} \operatorname{reg}\left(\mathscr{Y}_{n}\right)=-\infty$. Thus, $\lim _{n \rightarrow \infty} \operatorname{reg}\left(p^{*} \mathscr{Y}_{n}\right)=-\infty$ by Lemma 2.9. Since each $\mathscr{Y}_{n}$ is invertible away from a dimension- 0 set, $p^{*} \mathscr{Y}_{n}$ is invertible away from a locus of relative dimension 0. By Proposition 2.13, $\mathscr{F} \otimes_{X_{C}} p^{*} \mathscr{S}_{n}$ is 0-regular for $n \gg 0$. Theorem 2.10 shows that (1) and (2) of Definition 2.7 apply.

We now prove a uniform regularity result for certain subsheaves of a pullback of some $\mathscr{Y}_{n}$.

Lemma 6.2. There exists $m \geq 0$ so that the following holds for any $n \geq m$ : for any commutative noetherian $\mathbb{k}$-algebra $p: \mathbb{k} \rightarrow C$, for any $C$-point $y$ of $X$, and for any coherent sheaf $\mathscr{K}$ on $X_{C}$ so that $\mathscr{I}_{y} p^{*} \mathscr{Y}_{n} \subseteq \mathscr{K} \subseteq p^{*} \mathscr{Y}_{n}$, $\mathscr{K}$ is 0 -regular. In particular, $\mathscr{K}$ is globally generated and $R^{1} q_{*} \mathscr{H}=0$.

Proof. Let $D$ be the constant from Proposition 2.13, and let $r:=\operatorname{reg}\left(\mathrm{O}_{X}\right)$. By [Rogalski and Stafford 2007, Corollary 3.14], we have $\lim _{n \rightarrow \infty} \operatorname{reg}\left(\mathscr{Y}_{n}\right)=-\infty$. Let $m$ be such that for all $n \geq m, \operatorname{reg}\left(\mathscr{Y}_{n}\right) \leq-r-D-1$. We claim this $m$ satisfies the conclusions of the lemma.

Fix a commutative noetherian $\mathbb{k}$-algebra $p: \mathbb{k} \rightarrow C$ and a $C$-point $y$ of $X$. We first claim that $\operatorname{reg}\left(\Phi_{y}\right) \leq r+1$. To see this, let $i \geq 1$ and consider the exact sequence

$$
\begin{aligned}
R^{i-1} q_{*} \mathrm{O}_{X_{C}}(r+1-i) \stackrel{\alpha}{\rightarrow} R^{i-1} q_{*} \mathrm{O}_{y}(r+1-i) \\
\rightarrow R^{i} q_{*} \Phi_{y}(r+1-i) \rightarrow R^{i} q_{*} \mathcal{O}_{X_{C}}(r+1-i) .
\end{aligned}
$$

The last term vanishes as $\mathrm{O}_{X_{C}}$ is $(r+1)$-regular by Lemma 2.9 and Theorem 2.10(1). If $i \geq 2$, then $R^{i-1} q_{*} O_{y}(r+1-i)=0$ for dimension reasons, so $R^{i} q_{*} \Phi_{y}(r+1-i)=0$. On the other hand, if $i=1$, then because $0_{X_{C}}(r)$ is 0 -regular, by Lemma 2.11, $\alpha$ is surjective. Again, $R^{i} q_{*} \Phi_{y}(r+1-i)=0$. Thus, $\Phi_{y}$ is $(r+1)$-regular as claimed.

Let $n \geq m$. By Lemma 2.9, $\operatorname{reg}\left(p^{*} \mathscr{S}_{n}\right)=\operatorname{reg}\left(\mathscr{S}_{n}\right)$. Note that $\mathscr{I}_{y}$ and $p^{*} \mathscr{S}_{n}$ are both locally free away from a set of relative dimension 0 . Thus, the hypotheses of Proposition 2.13 apply, and by that result, we have

$$
\begin{aligned}
\operatorname{reg}\left(\Phi_{y} \otimes_{X_{C}} p^{*} \mathscr{S}_{n}\right) & \leq \operatorname{reg}\left(\mathscr{I}_{y}\right)+\operatorname{reg}\left(p^{*} \mathscr{S}_{n}\right)+D \\
& \leq r+1+D+\operatorname{reg}\left(p^{*} \mathscr{S}_{n}\right)=r+1+D+\operatorname{reg}\left(\mathscr{S}_{n}\right) .
\end{aligned}
$$

Our choice of $n$ ensures this is nonpositive. In particular, $\mathscr{I}_{y} \otimes_{X_{C}} p^{*} \mathscr{Y}_{n}$ is 0-regular.

Let $\mathscr{I}_{y} p^{*} \mathscr{Y}_{n} \subseteq \mathscr{K} \subseteq p^{*} \mathscr{Y}_{n}$. There is a natural map $f: \mathscr{I}_{y} \otimes_{X_{C}} p^{*} \mathscr{Y}_{n} \rightarrow \mathscr{K}$ given by the composition $\mathscr{I}_{y} \otimes_{X_{C}} p^{*} \mathscr{S}_{n} \rightarrow \mathscr{I}_{y} \cdot p^{*} \mathscr{Y}_{n} \subseteq \mathscr{K}$. The kernel and cokernel of $f$ are supported on a set of relative dimension 0 , and it is an easy exercise to show that $\mathscr{K}$ is therefore also 0 -regular. By Theorem 2.10, $\mathscr{K}$ is globally generated and $R^{1} q_{*} \mathscr{K}=0$ as claimed. 
Definition 6.3. We call a positive integer $m$ satisfying the conclusion of Lemma 6.2 a positivity parameter.

The proof of Lemma 6.2 shows that if we are willing to replace $\mathscr{L}$ by a sufficiently ample invertible sheaf, we may in fact assume that $m=1$ is a positivity parameter. (By [Keeler 2000, Theorem 1.2], the existence of a $\sigma$-ample sheaf means that any ample invertible sheaf is $\sigma$-ample.)

Corollary 6.4. Let $p: \mathbb{k} \rightarrow C$ be a noetherian commutative $\mathbb{k}$-algebra. Let $m$ be a positivity parameter (Definition 6.3), and let $n \geq m$.

(1) If $\mathscr{F} \subset p^{*} \mathscr{S}_{n}$ is a sheaf on $X_{C}$ so that $p^{*} \mathscr{Y}_{n} / \mathscr{F}$ has support on $X_{C}$ that is finite over $\operatorname{Spec}(C)$ and is a rank-1 projective $C$-module, then $q_{*} \mathscr{F}$ is a $C$-submodule of $q_{*} p^{*} \mathscr{Y}_{n}=S_{n} \otimes C$ such that the cokernel is rank-1 projective.

(2) If $\mathscr{K} \varsubsetneqq \mathscr{J} \subset p^{*} \mathscr{S}_{n}$ are sheaves on $X_{C}$ so that $p^{*} \mathscr{\varphi}_{n} / \mathscr{F}$ is a rank-1 projective $C$-module, then $q_{*} \mathscr{K} \varsubsetneqq q_{*} \mathscr{F}$.

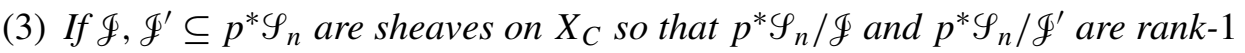
projective $C$-modules, then $q_{*} \mathscr{F}=q_{*} \mathscr{F}^{\prime}$ if and only if $\mathscr{F}=\mathscr{F}^{\prime}$.

Proof. (1) Let $x \in \operatorname{Spec} C$ be a closed point. Consider the fiber square

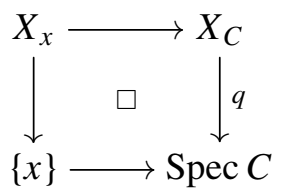

Let $\mathscr{F}_{x}:=\left.\mathscr{F}\right|_{X_{x}}$. Since $p^{*} \mathscr{S}_{n} / \mathscr{\mathscr { S }}$ is flat over Spec $C,\left.\mathscr{F}_{x} \subseteq p^{*} \mathscr{S}_{n}\right|_{X_{x}} \cong \mathscr{I}_{n} \otimes_{\mathbb{k}} \mathbb{k}(x)$. Further, $\left(\mathscr{S}_{n} \otimes_{\mathbb{k}} \mathbb{k}(x)\right) / \mathscr{F}_{x} \cong 0_{x}$. By our choice of $n$, therefore, $H^{1}\left(X_{x}, \mathscr{F}_{x}\right)=0$.

Now $\mathscr{F}$ is the kernel of a surjective morphism of flat sheaves and so is flat over Spec $C$. Since $H^{1}\left(X_{x}, \mathscr{F}_{x}\right)=0$, by the theorem on cohomology and base change [Hartshorne 1977, Theorem III.12.11(a)], we have $R^{1} q_{*} \mathscr{F}_{C} \otimes_{C}(x)=0$. The $C$-module $R^{1} q_{*} \mathscr{F}$ thus vanishes at every closed point and is therefore 0 .

The complex

$$
0 \rightarrow q_{*} \mathscr{I} \rightarrow q_{*} p^{*} \mathscr{S}_{n} \rightarrow q_{*}\left(p^{*} \mathscr{S}_{n} / \mathscr{F}\right) \rightarrow 0
$$

is thus exact. By assumption, $q_{*}\left(p^{*} \mathscr{Y}_{n} / \mathscr{F}\right)$ is a rank-1 projective $C$-module. Since cohomology commutes with flat base change [Hartshorne 1977, Proposition III.9.3], we have $q_{*} p^{*} \mathscr{S}_{n} \cong H^{0}\left(X, \mathscr{S}_{n}\right) \otimes_{\mathbb{k}} C=S_{n} \otimes_{\mathbb{k}} C$.

(2) Since $m$ is a positivity parameter, $\mathscr{F}$ is globally generated, and it follows immediately that $q_{*} \mathcal{H} \neq q_{*} \mathscr{F}$.

(3) From (2), we have

$$
q_{*}\left(\mathscr{F} \cap \mathscr{F}^{\prime}\right)=q_{*} \mathscr{F} \Longleftrightarrow \mathscr{F} \cap \mathscr{F}^{\prime}=\mathscr{F} \Longleftrightarrow \mathscr{F} \subseteq \mathscr{F}^{\prime} .
$$


It follows from our assumptions that this occurs if and only if $\mathscr{E}=\mathscr{F}^{\prime}$. Further,

$$
q_{*} \mathscr{F}=q_{*}\left(\mathscr{F} \cap \mathscr{F}^{\prime}\right)=q_{*}(\mathscr{F}) \cap q_{*} \mathscr{F}^{\prime} \Longleftrightarrow q_{*} \mathscr{F} \subseteq q_{*} \mathscr{F}^{\prime} .
$$

From (1), we obtain that this is equivalent to $q_{*} \mathscr{F}=q_{*} \mathscr{F}^{\prime}$.

We now apply these regularity results to show that ${ }_{l} Y_{\infty} \cong{ }_{l} Z_{\infty}$ for $l \gg 0$. We will need the following easy lemma:

Lemma 6.5. Let $A$ and $B$ be commutative noetherian local $\mathbb{k}$-algebras with residue field $\mathbb{k}$ and $s: A \rightarrow B$ a local homomorphism. If $s^{*}: \operatorname{Hom}^{\mathrm{alg}}(B, C) \rightarrow \operatorname{Hom}^{\mathrm{alg}}(A, C)$ is surjective for all finite-dimensional commutative local $\mathbb{k}$-algebras $C$, then $s$ is injective.

Proof. Let $\mathfrak{m}$ be the maximal ideal of $A$, and let $\mathfrak{n}$ be the maximal ideal of $B$. Let $f \in \operatorname{ker} s$. Suppose first that there is some $k$ so that $f \in \mathfrak{m}^{k-1} \backslash \mathfrak{m}^{k}$. Let $C:=A / \mathfrak{m}^{k}$, and let $\pi: A \rightarrow C$ be the natural map. Then $C$ is a finite-dimensional artinian local $\mathbb{k}$-algebra. Now, $\pi(f) \neq 0$ but $s(f)=0$. Thus, $\pi \notin \operatorname{Im}\left(s^{*}\right)$, a contradiction.

We thus have ker $s \subseteq \bigcap_{k} \mathfrak{m}^{k}$. By the Artin-Rees lemma, ker $s=0$.

Proposition 6.6. Let $m$ be a positivity parameter, and let $n \geq l \geq m$. Let ${ }_{l} Z_{n}$ be the l-shifted length- $(n-l+1)$ point scheme of $\mathscr{S}$ with truncation morphism $\phi_{n}: Z_{n} \rightarrow Z_{n-1}$ as in Proposition 5.9. Let ${ }_{l} Y_{n}$ be the l-shifted length- $(n-l+1)$ point scheme of $S$ with truncation morphism $\chi_{n}:{ }_{l} Y_{n} \rightarrow{ }_{l} Y_{n-1}$ as in Theorem 5.11. Let ${ }_{l} Z_{\infty}:=\lim _{l} Z_{n}$, and let ${ }_{l} Y_{\infty}:=\lim _{\leftarrow} Y_{n}$.

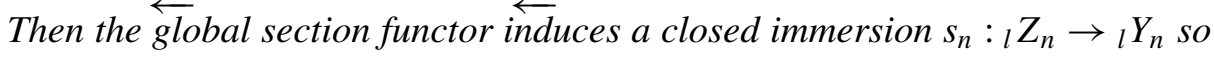
that the following diagram commutes:

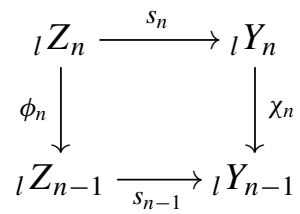

Proof. Let $p: \mathbb{k} \rightarrow C$ be a commutative noetherian $\mathbb{k}$-algebra. Let $p: X_{C} \rightarrow X$ and $q: X_{C} \rightarrow \operatorname{Spec} C$ be the two projection maps as usual. Note that if $\mathscr{S} \subset p^{*} \mathscr{Y}_{\geq l}$ is the defining ideal of an $l$-shifted length- $(n-l+1)$ truncated $C$-point module over $\mathscr{S}$, then by Corollary 6.4(1), $q_{*} \mathscr{F}$ is the defining ideal of an $l$-shifted length- $(n-l+1)$ truncated $C$-point module over $S$. Thus, $s_{n}$ is well defined. By Corollary 6.4(3), $s_{n}$ is injective on $\mathbb{k}$-points and on $\mathbb{k}[\epsilon]$ points. It is standard [Harris 1992, proof of Theorem 14.9] that, because $s_{n}$ is projective, $s_{n}$ is a closed immersion. That (6.7) commutes is immediate.

We will see that ${ }_{l} Y_{\infty}$ and ${ }_{l} Z_{\infty}$ are isomorphic. It does not seem to be generally true that ${ }_{l} Y_{n}$ and ${ }_{l} Z_{n}$ are isomorphic, but we will see that there is an induced isomorphism of certain naturally defined closed subschemes. 
Let $l \in \mathbb{N}$. For any $n$, let $\Phi_{n}:{ }_{l} Z_{\infty} \rightarrow{ }_{l} Z_{n}$ and $\Upsilon_{n}:{ }_{l} Y_{\infty} \rightarrow{ }_{l} Y_{n}$ be the induced maps. For any $n \geq l$, define ${ }_{l} Z_{n}^{\prime} \subseteq{ }_{l} Z_{n}$ to be the image of $\Phi_{n}:{ }_{l} Z_{\infty} \rightarrow{ }_{l} Z_{n}$. That is,

$$
{ }_{l} Z_{n}^{\prime}=\bigcap_{i \geq 0} \operatorname{Im}\left(\phi^{i}:{ }_{l} Z_{n+i} \rightarrow{ }_{l} Z_{n}\right) .
$$

Since ${ }_{l} Z_{n}$ is noetherian and the $\phi_{n}$ are closed, this is a closed subscheme of ${ }_{l} Z_{n}$ equal to $\operatorname{Im}\left(\phi^{k}:{ }_{l} Z_{n+k} \rightarrow{ }_{l} Z_{n}\right)$ for some $k$. Similarly, let ${ }_{l} Y_{n}^{\prime}=\operatorname{Im}\left(\Upsilon_{n}:_{l} Y_{\infty} \rightarrow{ }_{l} Y_{n}\right)$. Clearly ${ }_{l} Z_{\infty}=\lim _{l} Z_{n}^{\prime}$ and ${ }_{l} Y_{\infty}=\lim _{l} Y_{n}^{\prime}$. We refer to ${ }_{l} Z_{n}^{\prime}$ and ${ }_{l} Y_{n}^{\prime}$ as essential point schemes as modules in ${ }_{l} Z_{n}^{\prime}$ and ${ }_{l} Y_{n}^{\prime}$ are truncations of honest (shifted) point modules.

Theorem 6.8. Let $m$ be a positivity parameter, and let $n \geq l \geq m$.

(1) The morphism $s_{n}:{ }_{l} Z_{n} \rightarrow{ }_{l} Y_{n}$ defined in Proposition 6.6 induces an isomorphism of essential point schemes

$$
s_{n}^{\prime}:{ }_{l} Z_{n}^{\prime} \stackrel{\cong}{\rightarrow} Y_{n}^{\prime} .
$$

(2) The limit $s:{ }_{l} Z_{\infty} \rightarrow{ }_{l} Y_{\infty}$ is an isomorphism of stacks.

Proof. Since the subscript $l$ will remain fixed, we suppress it in the notation. Let $s_{n}^{\prime}:=\left.s_{n}\right|_{Z_{n}^{\prime}}$. It follows from commutativity of (6.7) that $s_{n}^{\prime}\left(Z_{n}^{\prime}\right) \subseteq Y_{n}^{\prime}$.

We next prove (2). The limit $s=\lim _{\leftarrow} s_{n}$ is clearly a morphism of stacks, that is, a natural transformation of functors. Let $C$ be a commutative finite-dimensional local $\mathbb{k}$-algebra. We will show that $s$ is bijective on $C$-points.

Let $y \in Y_{\infty}(C)$ be a $C$-point of $Y_{\infty}$, which by Theorem 5.11 corresponds to an exact sequence

$$
0 \rightarrow J \rightarrow\left(S_{C}\right)_{\geq l} \rightarrow M \rightarrow 0,
$$

where $M$ is an $l$-shifted $S_{C}$-point module.

For $i \geq l$, let $\mathscr{F}_{i} \subseteq p^{*} \mathscr{S}_{i}$ be the subsheaf generated by $J_{i} \subseteq q_{*} p^{*} \mathscr{S}_{i}$. Let $\mathscr{g}:=$ $\bigoplus_{i \geq l} \mathscr{F}_{i}$. We will show that $\mathcal{M}:=p^{*} \mathscr{Y}_{\geq l} / \mathscr{\mathscr { F }}$ is an $l$-shifted $p^{*} \mathscr{S}$-point module, that is, that there is a $C$-point $y$ of $X$ so that $\mathcal{M}_{n} \cong O_{y} \otimes p^{*} \mathscr{L}_{n}$ for all $n \geq l$.

Since $p^{*} \mathscr{G}_{j}$ is globally generated for $j \geq m$, we have $\mathscr{F}_{i} p^{*} \mathscr{Y}_{j}^{\sigma_{j}^{i}} \subseteq \mathscr{F}_{i+j}$ for $i \geq l$ and $j \geq m$. Therefore, $M$ is a coherent right module over the bimodule algebra $\mathscr{Y}^{\prime}:=\mathcal{O}_{X_{C}} \oplus \bigoplus_{j \geq m} p^{*} \mathscr{Y}_{j}$. Further, each $\mathcal{M}_{j}$ is clearly torsion over $X$ as $\operatorname{Spec} C$ is zero-dimensional. As in [Rogalski and Stafford 2007, Lemma 4.1(1)], it follows from critical density of the orbits of the points in $P$ that there are a coherent $X$ torsion sheaf $\mathscr{F}$ on $X_{C}$ and $n_{0} \geq l$ so that $M_{j} \cong \mathscr{F}_{F} \otimes_{X_{C}} p^{*} \mathscr{L}_{j}$ for $j \geq n_{0}$. By critical density again, there is $n_{1} \geq n_{0}$ so that

$$
\operatorname{Supp}(\mathscr{F}) \cap p^{-1}\left(\sigma^{-j}(P)\right)=\varnothing
$$

for $j \geq n_{1}$. This implies that for $j \geq n_{1}$ and $k \geq 1$, we have

$$
\mu_{j} \cdot p^{*} \mathscr{G}_{k}^{\sigma^{j}}=\mathcal{M}_{j} \otimes_{X_{C}} p^{*} \mathscr{G}_{k}^{\sigma^{j}}=\mathcal{M}_{j} \otimes_{X_{C}} p^{*} \mathscr{L}_{k}^{\sigma^{j}} \cong \mathcal{M}_{j+k}
$$


and $\mathscr{F}_{j}\left(p^{*} \mathscr{Y}_{k}^{\sigma^{j}}\right)=\mathscr{F}_{j+k}$. (In particular, $\mathscr{F}_{\geq n_{1}}$ and $\mathcal{M}_{\geq n_{1}}$ are right $p^{*} \mathscr{S}_{\text {-modules.) }}$

By Lemma 6.1, $\left\{p^{*} \mathscr{Y}_{k}^{\sigma^{j}}\right\}_{k \geq 0}$ is right ample on $X_{C}$. As in [Rogalski and Stafford 2009, Lemma 9.3], for any $i \geq l$ and $k \gg 0$, we have $q_{*}\left(\mathscr{\mathscr { F }}_{i} p^{*} \mathscr{G}_{k}^{\sigma^{i}}\right)=J_{i}\left(S_{C}\right)_{k} \subseteq J_{i+k}$. For $i \geq n_{1}$ and for $k \geq 1$, we have $q_{*}\left(\mathscr{F}_{i} p^{*} \mathscr{G}_{k}^{\sigma^{i}}\right)=q_{*} \mathscr{F}_{i+k} \supseteq J_{i+k}$. There is thus $n_{2} \geq n_{1}$ so that $J_{j}=q_{*} \mathscr{F}_{j}$ for $j \geq n_{2}$.

It follows from Lemma 6.1 that there is $n_{3} \geq n_{2}$ so that the top row of

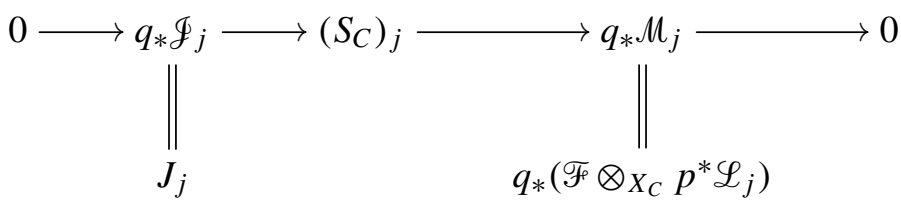

is exact for $j \geq n_{3}$. Thus, $q_{*}\left(\mathscr{F}_{F} \otimes_{X_{C}} p^{*} \mathscr{L}_{j}\right) \cong\left(S_{C}\right)_{j} / J_{j} \cong C$ for $j \geq n_{3}$. Since $\left\{p^{*} \mathscr{L}_{j}\right\}$ is right ample, this implies that $\mathscr{F}_{F} \cong \mathscr{O}_{y}$ for some $C$-point $y$ of $X$.

For $l \leq i \leq n_{3}$, let $\mathscr{F}_{i}^{\prime}:=\mathscr{F}_{i}+\mathscr{I}_{y} p^{*} \mathscr{Y}_{i}$. By choice of $l, \mathscr{F}_{i}^{\prime}$ is 0 -regular. Thus, the rows of the commutative diagram

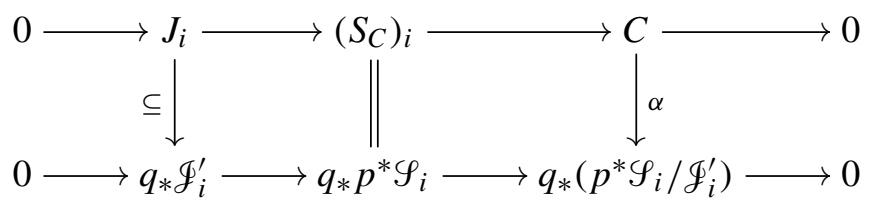

are exact, and $\alpha$ is therefore surjective. This shows that, as a $\left(C \cong O_{y}\right)$-module, $p^{*} \mathscr{Y}_{i} / \mathscr{F}_{i}^{\prime}$ is cyclic.

Let $N:=\operatorname{Ann}_{C}\left(p^{*} \mathscr{Y}_{i} / \mathscr{F}_{i}^{\prime}\right)$. For $i \gg 0, p^{*} \mathscr{S}_{i} / \mathscr{I}_{i} \cong \mathcal{O}_{y} \otimes_{X_{C}} p^{*} \mathscr{L}_{i}$ is killed by $\mathscr{I}_{y}$. Thus, for $i \gg 0$, we have $\left(\mathscr{F}_{i}^{\prime}\right)_{y}=\left(\mathscr{F}_{i}\right)_{y}+\left(\mathscr{I}_{y} p^{*} \mathscr{G}_{i}\right)_{y}=\left(\mathscr{F}_{i}\right)_{y}$, and

$$
\begin{aligned}
\left(\mathscr{F}_{i+j}\right)_{y} \supseteq\left(\mathscr{F}_{i}\right)_{y}\left(p^{*} \mathscr{S}_{j}^{\sigma^{i}}\right)_{y} & =\left(\mathscr{F}_{i}^{\prime}\right)_{y}\left(p^{*} \mathscr{S}_{j}^{\sigma^{i}}\right)_{y} \\
& \supseteq N\left(p^{*} \mathscr{Y}_{i}\right)_{y}\left(p^{*} \mathscr{Y}_{j}^{\sigma^{i}}\right)_{y}=N\left(p^{*} \mathscr{Y}_{i+j}\right)_{y} .
\end{aligned}
$$

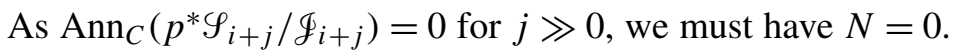

Thus, in fact $p^{*} \mathscr{Y}_{i} / \mathscr{F}_{i}^{\prime} \cong \mathcal{O}_{y} \cong C$. Looking again at (6.9), we see that $\alpha$ is an isomorphism, and so $J_{i}=q_{*} \mathscr{F}_{i}^{\prime}$. As $\mathscr{F}_{i}^{\prime}$ is 0-regular, it is globally generated: in other words, $\mathscr{I}_{i}=\mathscr{F}_{i}^{\prime}$.

We still need to show $\mathscr{g}$ is a $p^{*} \mathscr{S}$-module. Let $i \geq l$, and suppose $\mathscr{F}_{i} p^{*} \mathscr{S}_{1}^{\sigma^{i}} \nsubseteq \mathscr{F}_{i+1}$, so $\left(\mathscr{F}_{i+1}+\mathscr{F}_{i} p^{*} \mathscr{G}_{1}^{\sigma^{i}}\right) / \mathscr{F}_{i+1}$ is a nonzero submodule of $p^{*} \mathscr{Y}_{i+1} / \mathscr{\mathscr { F }}_{i+1} \cong \mathscr{O}_{y}$. Since $\left(\mathscr{F}_{i+1}+\mathscr{F}_{i} p^{*} \mathscr{Y}_{1}^{\sigma^{i}}\right) \mathscr{S}_{j}^{\sigma^{i+1}} \subseteq \mathscr{F}_{i+j+1}$ for all $j \gg 0$, a similar argument to the last paragraph but one produces a contradiction.

Thus, $p^{*} \mathscr{S}_{\geq l} / \mathscr{F}$ is an $l$-shifted $C$-point module for $\mathscr{Y}$, and $q_{*} \mathscr{F}=J$. This shows that $s: Z_{\infty} \rightarrow Y_{\infty}$ induces a surjection on $C$-points. It follows from Corollary 6.4(3) that $s$ is injective on $C$-points. 
Consider the commutative diagram

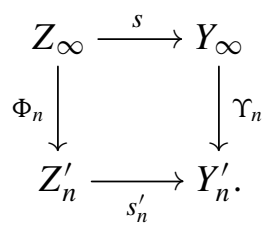

Let $x \in X$ be a k-point. There is some $N$ so that for $n \geq N, \Phi_{n}$ is a local isomorphism at all points in the preimage of $x$. We claim that for $n \geq N$, in fact all maps in (6.10) are local isomorphisms at all points in the preimage of $x$. In particular, $s$ is an isomorphism in the preimage of $x$; since $x$ was arbitrary, $s$ is therefore an isomorphism of stacks.

So it suffices to prove the claim. We may work locally. If $z \in Z_{\infty}$ is a point lying over $x$, let $z_{n}, y$, and $y_{n}$ be the images of $z$ in $Z_{n}^{\prime}, Y_{\infty}$, and $Y_{n}^{\prime}$, respectively. Let $B:=\mathfrak{O}_{Z_{\infty}, z} \cong \mathcal{O}_{Z_{n}^{\prime}, z_{n}}$. Let $A:=\mathfrak{O}_{Y_{\infty}, y}$, and let $A^{\prime}:=\mathcal{O}_{Y_{n}^{\prime}, y_{n}}$. We have a commutative diagram of local homomorphisms

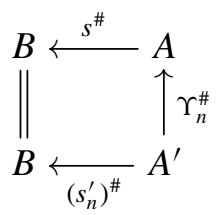

As $\Upsilon_{n}$ is scheme-theoretically surjective, $\Upsilon_{n}^{\#}$ is injective. It follows from Proposition 5.5 that $A$ is isomorphic to a local ring of some $Y_{m}^{\prime}$ and in particular is a noetherian $\mathbb{k}$-algebra. By Lemma $6.5, s^{\#}$ is injective. Thus, $\left(s_{n}^{\prime}\right)^{\#}$ is injective. As $s_{n}$ is a closed immersion, $\left(s_{n}^{\prime}\right)^{\#}$ is also surjective and thus an isomorphism; thus, all maps in (6.10) are local isomorphisms above $x$. This proves the claim as required.

We now prove (1). Consider the diagram (6.10). By Proposition 6.6, $s_{n}: Z_{n} \rightarrow Y_{n}$ is a closed immersion. Thus, the restriction $s_{n}^{\prime}: Z_{n}^{\prime} \rightarrow Y_{n}^{\prime}$ is also a closed immersion.

On the other hand, $Y_{n}^{\prime}$ is the scheme-theoretic image of $\Upsilon_{n}$ and $s$ is an isomorphism. Thus, the composition $\Upsilon_{n} s=s_{n}^{\prime} \Phi_{n}$ is scheme-theoretically surjective, so $s_{n}^{\prime}$ is scheme-theoretically surjective. But a scheme-theoretically surjective closed immersion is an isomorphism.

Of course, the defining ideal of a 1 -shifted point module also defines a 0 -shifted point module, so if the positivity parameter $m=1$, the conclusions of Theorem 6.8 in fact hold for $m=0$. In this situation, we will refer to $m=0$ as a positivity parameter, by slight abuse of notation, since we need only the isomorphism ${ }_{l} Y_{\infty} \cong{ }_{l} Z_{\infty}$ for $l \geq m$ in the sequel.

Corollary 6.11. Let $l \in \mathbb{N}$, and let ${ }_{l} Y_{\infty}$ be the moduli stack of embedded $l$-shifted $S$-point modules as above. Then ${ }_{l} Y_{\infty}$ is a noetherian fpqc-algebraic stack. 
Proof. We know that ${ }_{l} Y_{\infty}$ is a sheaf in the fpqc topology by Theorem 5.11. Let $m$ be a positivity parameter. If $l \geq m$, then ${ }_{l} Y_{\infty} \cong{ }_{l} Z_{\infty}$ is noetherian fpqc-algebraic by Theorem 5.11.

Suppose then that $l<m$. Let $T:{ }_{l} Y_{\infty} \rightarrow{ }_{m} Y_{\infty}$ be the morphism defined by $T(M):=M_{\geq m}$. It is straightforward that $T$ is a morphism of functors and the product

$$
\Phi_{m} \times T:{ }_{l} Y_{\infty} \rightarrow{ }_{l} Y_{m} \times{ }_{X}\left({ }_{m} Y_{\infty}\right)
$$

is a closed immersion. Since ${ }_{m} Y_{\infty}$ is noetherian by the first paragraph, it has an fpqc cover $U \rightarrow{ }_{m} Y_{\infty}$ by a noetherian affine scheme $U$. This cover can clearly be lifted and refined to induce an fpqc cover $V \rightarrow{ }_{l} Y_{m} \times{ }_{X}\left({ }_{m} Y_{\infty}\right)$ where $V$ is a noetherian affine scheme. But then the Cartesian product

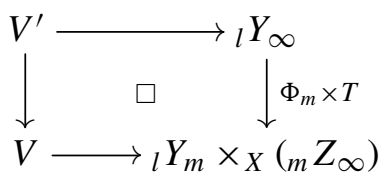

gives an fpqc cover $V^{\prime} \rightarrow Y_{\infty}$. Since $\Phi_{m} \times T$ is a closed immersion, so is $V^{\prime} \rightarrow V$. Thus, $V^{\prime}$ is isomorphic to a closed subscheme of $V$ and is noetherian and affine.

Let $m$ be a positivity parameter, and let $l \geq m$. We note that the relevant component of $Y_{\infty} \cong{ }_{l} Z_{\infty}$ is the component containing the $\mathbb{k}(X)$-point corresponding to the generic point module $\mathbb{k}(X) z^{l} \oplus \mathbb{k}(X) z^{l+1} \oplus \cdots$, which is isomorphic to $\left(Q_{\mathrm{gr}}(S)\right)_{\geq l}$.

\section{A coarse moduli space for point modules}

In this section, we consider point modules up to module isomorphism in qgr- $S$ and show that the scheme $X$ is a coarse moduli scheme for this functor.

We define the following maps. For any $l$, let $\Phi:{ }_{l} Z_{\infty} \rightarrow X$ be the map induced from the $f_{n}$. Let $\Psi:{ }_{0} Z_{\infty} \rightarrow{ }_{0} Z_{\infty}$ be the map induced from $\psi_{n}:{ }_{0} Z_{n} \rightarrow{ }_{0} Z_{n-1}$. Taking the limit of (5.8), we obtain that

$$
\Phi \Psi=\sigma \Phi:{ }_{0} Z_{\infty} \rightarrow X .
$$

For any noetherian commutative $\mathbb{k}$-algebra $C$, there is a graded $\left(\mathbb{O}_{X_{C}}, \sigma \times 1\right)$ bimodule algebra $\mathscr{S}_{C}$ given by pulling back $\mathscr{S}$ along the projection map $X_{C} \rightarrow X$. Taking global sections gives a functor $\mathrm{Gr}-\mathscr{S}_{C} \rightarrow \mathrm{Gr}-S_{C}$. If $C=\mathbb{k}$, this induces an equivalence qgr- $\mathscr{S}_{C} \rightarrow$ qgr- $S_{C}$ by Theorem 2.8. In order to avoid the issues involved with extending this result to bimodule algebras over arbitrary base schemes, we work instead with point modules in Qgr- $\mathscr{S}_{C}$ and Qgr- $S_{C}$.

Let $l \geq m$, where $m$ is a positivity parameter (Definition 6.3), and let $F$ be the moduli functor of (embedded) $l$-shifted point modules over $S$ as in the previous section. Define an equivalence relation $\sim$ on $F(C)$ by saying that $M \sim N$ if their images are 
isomorphic in Qgr- $S_{C}$. Define a contravariant functor $G:$ Affine schemes $\rightarrow$ Sets by sheafifying, in the fpqc topology, the presheaf $G^{\text {pre }}$ of sets Spec $C \mapsto F(C) / \sim$. Let $\mu: F \rightarrow G$ be the natural map, $\mathscr{F} \cong{ }_{l} Z_{\infty}$ the moduli functor of $l$-shifted point modules over $\mathscr{Y}$, and $\mathscr{G}:=\mathscr{F} / \sim$ as above. Let $\mu: \mathscr{F} \rightarrow \mathscr{G}$ be the natural map.

We recall that $a_{n}: X_{n} \rightarrow X$ is the blowup of $X$ at $\Phi_{n}$ and that by Corollary 3.3 there are morphisms $\alpha: X_{n+1} \rightarrow X_{n}$ that intertwine with the maps $a_{n}$.

We briefly discuss point modules over local rings. We note that if $C$ is a local ring of a point of $Z_{\infty}^{o} \cong X_{\infty}$ with maximal ideal $\mathfrak{m}$, then the map $h_{Z_{\infty}}(C) \rightarrow \mathscr{F}(C)$ has a particularly simple form. Let $\zeta$ : Spec $C \rightarrow X_{\infty}$ be the induced morphism. By critical density, there is some $n \geq m$ so that $\zeta_{n}(\mathfrak{m})$ is not a fundamental point of any of the maps $\alpha^{i}: X_{i+n} \rightarrow X_{n}$ for any $i>0$. Let $x_{n}:=\zeta_{n}(\mathfrak{m})$. Define

$$
a_{n}^{-1}\left(\mathscr{Y}_{j}\right):=a_{n}^{-1}\left(\mathscr{\Phi}_{j}\right) \otimes_{X_{n}} a_{n}^{*} \mathscr{L}_{j} .
$$

Then $a_{n}^{-1}\left(\mathscr{Y}_{j}\right)$ is flat at $x_{n}$ for all $j$. Let

$$
\mathcal{M}:=\bigoplus_{j \geq 0} a_{n}^{-1}\left(\mathscr{Y}_{j}\right)_{x_{n}} .
$$

Then $M$ is flat over $C$ and is the $C$-point module corresponding to $\zeta$. The $C$-action on $\mathcal{M}$ is obvious; to define the $\mathscr{S}$-action on $\mathcal{M}$, let $x:=a_{n}\left(x_{n}\right)$. Then there are maps

$$
\mu_{j} \otimes_{\mathbb{k}} \mathscr{Y}_{i}(X) \rightarrow \mu_{j} \otimes_{\mathbb{k}} \mathscr{Y}_{i}^{\sigma^{j}}(X) \rightarrow \mu_{j} \otimes_{\mathcal{O}_{X, x}}\left(\mathscr{S}_{i}^{\sigma^{j}}\right)_{x} \rightarrow a_{n}^{-1}\left(\mathscr{S}_{j+i}\right)_{x_{n}} .
$$

This gives a right $\mathscr{S}$-action on $\mathcal{M}$; by letting $C$ act naturally on the left and identifying $C$ with $C^{\text {op }}$, we obtain an action of $\mathscr{S}_{C}$ on $M$.

If $C$ is a local ring, we do not know if $S_{C}$ is necessarily noetherian. However, $C$-point modules in Qgr- $S_{C}$ are well behaved as follows.

Lemma 7.1. Let $C$ be a commutative noetherian local $\mathbb{k}$-algebra. Let $N$ and $M$ be l-shifted $C$-point modules with $M \cong N$ in $\mathrm{Qgr}-S_{C}$. Then for some $k$, we have $M_{\geq k} \cong N_{\geq k}$.

Proof. The torsion submodules of $M$ and $N$ are trivial. Thus,

$$
\operatorname{Hom}_{\mathrm{Qgr}-S_{C}}(M, N)=\underset{\lim }{\longrightarrow} \operatorname{Hom}_{\mathrm{Gr}-S_{C}}\left(M^{\prime}, N\right),
$$

where the limit is taken over all submodules $M^{\prime} \subseteq M$ with $M / M^{\prime}$ torsion. If $M \cong N$ in Qgr- $S_{C}$, then there is some submodule $M^{\prime} \subseteq M$ so that $M / M^{\prime}$ is torsion and so that there is a homomorphism $f: M^{\prime} \rightarrow N$ so that ker $f$ and $N / f\left(M^{\prime}\right)$ are torsion. Since $M$ and $N$ are torsion-free, we must have ker $f=0$.

Thus, it suffices to show that if $N$ is an $l$-shifted $C$-point module and $M \subseteq N$ is a graded submodule with $T:=N / M$ torsion, then $T_{n}=0$ for $n \gg 0$. 
Let $L$ be the residue field of $C$. By assumption, $N$ is $C$-flat. Thus, there is an exact sequence

$$
0 \rightarrow \operatorname{Tor}_{1}^{C}(T, L) \rightarrow M_{L} \rightarrow N_{L} \rightarrow T_{L} \rightarrow 0 .
$$

By [Artin et al. 1999, Theorem 5.1], the algebra $R_{L}$ is noetherian. Thus, $N_{L}$ and $T_{L}$ are also noetherian. Since $T_{L}$ is torsion, it is finite-dimensional. Thus, for $n \gg 0$, we have $\left(T_{L}\right)_{n}=\left(T_{n}\right) \otimes_{C} L=0$. By Nakayama's lemma, $T_{n}=0$.

Lemma 7.2. Let $C$ be a noetherian local ring, and let $M$ and $\mathcal{N}$ be $C$-point modules over $\mathscr{Y}$, corresponding to morphisms $f_{\mathcal{M}}, f_{\mathcal{N}}: \operatorname{Spec} C \rightarrow{ }_{0} Z_{\infty}$. If $\mathcal{N} \sim \mathcal{M}$, then there is some $k$ so that $\Psi^{k} f_{\mathcal{M}}=\Psi^{k} f_{\mathcal{N}}$.

Proof. Let $m$ be a positivity parameter, and let $M:=s\left(\mathcal{M}_{\geq m}\right)$ and $N:=s\left(\mathcal{N}_{\geq m}\right)$. Then $M \sim N$; by Lemma 7.1, there is some $k$, which we may take to be at least $m$, so that $M_{\geq k} \cong N_{\geq k}$. Since ${ }_{k} Z_{\infty} \cong{ }_{k} Y_{\infty}$, we have $M_{\geq k} \cong \mathcal{N}_{\geq k}$. Thus, the modules $\mathcal{M}[k]_{\geq 0} \cong \mathcal{N}[k]_{\geq 0}$ correspond to the same point of ${ }_{0} Z_{\infty}$; that is, $\Psi^{k} f_{\mathcal{M}}=\Psi^{k} f_{\mathcal{N}}$.

We now show that $X$ is a coarse moduli space for ${ }_{0} Z_{\infty} / \sim$. In fact, we prove this result in greater generality to be able to analyze the spaces ${ }_{l} Y_{\infty}$.

Proposition 7.3. Let $Z_{\infty}:={ }_{0} Z_{\infty}$. Let $V_{\infty}$ be a closed algebraic substack of $Z_{\infty}$ so that $X_{\infty} \subseteq V_{\infty} \subseteq Z_{\infty}$, and assume that $V_{\infty}=\lim V_{n}$, where $V_{n} \subset Z_{n}$ is a closed subscheme that maps into $V_{n-1}$ under $Z_{n} \rightarrow \overleftarrow{Z_{n-1}}$ for all $n$. Then $X$ is a coarse moduli space for $V_{\infty} / \sim$. More precisely, let $\mathcal{H}$ be the image of $V_{\infty}$ under $\mu: Z_{\infty} \rightarrow$ G. Then

(1) the morphism $\Phi: V_{\infty} \rightarrow X$ factors via $V_{\infty} \stackrel{\mu}{\rightarrow} \mathscr{H} \stackrel{t}{\rightarrow} X$ and

(2) every morphism $\mathscr{H} \rightarrow A$ where $A$ is a scheme (of finite type) factors uniquely through $\mathscr{H} \stackrel{t}{\rightarrow} X$.

Proof. (1) It suffices to prove that if $C$ is a commutative noetherian ring and $M \sim \mathcal{N}$ are $C$-point modules over $\mathscr{Y}$, corresponding to maps $f_{\mathcal{M}}, f_{\mathcal{N}}: \operatorname{Spec} C \rightarrow Z_{\infty}$, then we have $\Phi f_{\mathcal{M}}=\Phi f_{\mathcal{N}}:$ Spec $C \rightarrow X$. To show this, it suffices to consider the case that $C$ is local. By Lemma 7.2, $\Psi^{k} f_{\mathcal{M}}=\Psi^{k} f_{\mathcal{N}}$ for some $k$. Thus, as required,

$$
\Phi f_{\mathcal{M}}=\sigma^{-k} \Phi \Psi^{k} f_{\mathcal{M}}=\sigma^{-k} \Phi \Psi^{k} f_{\mathcal{N}}=\Phi f_{\mathcal{N}} .
$$

(2) Let $v: \mathscr{H} \rightarrow h_{A}$ be a natural transformation for some scheme $A$. For all $n \in \mathbb{N}$, let $P_{n}$ be the subscheme of $X$ defined by $\Phi_{n}$. Fix any closed point $x \in X \backslash \bigcup P_{n}$; some such $x$ exists since $\mathbb{k}$ is uncountable. Let $C:=\mathscr{O}_{X, x}$. The induced map Spec $C \rightarrow X_{\infty} \rightarrow V_{\infty}$ gives a $C$-point module $\mu_{x}$ as described above; its $\sim$ equivalence class is an element of $\mathscr{H}(C)$. Applying $v$, we therefore have a morphism Spec $C \rightarrow A$. This extends to a morphism $g_{x}: U_{x} \rightarrow A$ for some open subset $U_{x}$ of $X$. It follows from critical density of the orbits of points in $P$ that $X \backslash \bigcup P_{n}$ 
is quasicompact. Thus, we may take finitely many $U_{x}$, say $U_{1}, \ldots, U_{k}$, that cover $X \backslash \bigcup P_{n}$ with maps $g_{i}: U_{i} \rightarrow A$. These maps all agree on the generic point of $X$ and so agree on overlaps $U_{i} \cap U_{j}$.

Let $U:=\bigcup_{i=1}^{k} U_{i}$, and let $g: U \rightarrow A$ be the induced map. Then $X \backslash U \subseteq \bigcup P_{n}$ is a closed subset of $X$, and so $X \backslash U=\left\{z_{1}, \ldots, z_{r}\right\}$ for some $z_{1}, \ldots, z_{r} \in \bigcup P_{n}$. Let $n$ be such that for any $i>0$, the map $\phi^{i}: Z_{n+i} \rightarrow Z_{n}$ is a local isomorphism at all points in the preimage of $\left\{z_{1}, \ldots, z_{r}\right\}$.

Let $\Phi_{n}: Z_{\infty} \rightarrow Z_{n}$ be the map induced from the $\phi_{m}$. There is an induced map $f_{n}^{-1}(U) \cap V_{n} \rightarrow U \rightarrow A$. Further, for every $y \in V_{\infty} \backslash \Phi^{-1}(U)$, the map Spec $\mathrm{O}_{V_{\infty}, y} \rightarrow V_{\infty} \rightarrow \mathscr{H} \rightarrow A$ factors through $V_{\infty} \rightarrow V_{n}$ as $\Phi_{n}$ is a local isomorphism at $y$. We thus obtain morphisms $\operatorname{Spec} \mathcal{O}_{V_{n}, y} \rightarrow A$ for all $y \in V_{n}$. Using these, we may extend $g$ to give a morphism $\theta: V_{n} \rightarrow A$ so that the following diagram commutes:

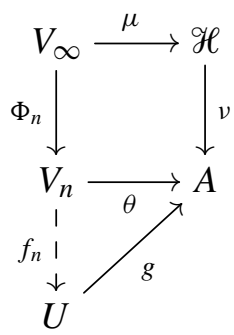

We claim that $\theta$ contracts each of the loci $f_{n}^{-1}\left(z_{j}\right) \cap V_{n}$ to a point. To see this, let $x, y \in \Phi^{-1}\left(z_{j}\right) \cap V_{\infty}$, corresponding to maps $f_{x}, f_{y}:$ Spec $\mathbb{k} \rightarrow V_{\infty}$. We must show that $\theta \Phi_{n} f_{x}=\theta \Phi_{n} f_{y}$.

Since for $k \gg 0, \sigma^{k}\left(z_{j}\right)$ is not in $\bigcup P_{n}, \Phi$ is a local isomorphism at $\Psi^{k}\left(\Phi^{-1}\left(z_{j}\right)\right)$. We have

$$
\Phi \Psi^{k} f_{x}=\sigma^{k} \Phi f_{x}=\sigma^{k} \Phi f_{y}=\Phi \Psi^{k} f_{y}
$$

and so $\Psi^{k} f_{x}=\Psi^{k} f_{y}$. Therefore, $\mu f_{x}=\mu f_{y}$, and so

$$
\theta \Phi_{n} f_{x}=v \mu f_{x}=v \mu f_{y}=\theta \Phi_{n} f_{y}
$$

as we wanted.

The morphism $\theta: V_{n} \rightarrow A$ thus factors set-theoretically to give a map from $X$ to $A$. Since $X$ is smooth at all $z_{i}$ by critical density of the orbits of the $z_{i}$, it is well known that $\theta$ also factors scheme-theoretically.

Consequently, we have the morphism $X \rightarrow A$ that we sought. This proves Proposition 7.3.

Theorem 7.4. Fix a positivity parameter $m$ (Definition 6.3), and let $l \geq m$. Then $X$ is a coarse moduli space for $G=F / \sim$.

Proof. As above, we let $\varphi$ denote the functor of $l$-shifted point modules over $\mathscr{S}$ modulo $\sim$. By Theorem 6.8(2), it is enough to show that $X$ is a coarse moduli 
space for $\mathscr{G}$. Let $L:{ }_{l} Z_{\infty} \rightarrow{ }_{0} Z_{\infty}$ be the map that sends $M \mapsto M[l]$. Notice that if $\mathcal{M}$ and $\mathcal{N}$ are $l$-shifted point modules, then $\mathcal{M} \sim \mathcal{N}$ if and only if $\mathcal{M}[l] \sim \mathcal{N}[l]$. That is, if we let $\mathscr{G}^{\prime}$ be the functor of (unshifted) point modules over $\mathscr{Y}$ modulo $\sim$, then $L$ induces an inclusion $G \rightarrow G^{\prime}$ so that the diagram

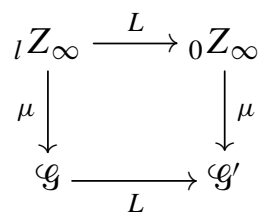

commutes. Let $V_{n}:=\operatorname{Im}\left({ }_{l} Z_{l+n} \rightarrow{ }_{0} Z_{n}\right)$ and $V_{\infty}:=\lim V_{n}$, so $V_{\infty}=L\left({ }_{l} Z_{\infty}\right)$.

Note that $L$ is injective on $X_{\infty} \subseteq{ }_{l} Y_{\infty}$. Thus, $V_{\infty}$ satisfies the hypotheses of Proposition 7.3, so $X$ is a coarse moduli scheme for $\mathscr{H}=\mu\left(V_{\infty}\right) \cong \mu\left({ }_{l} Z_{\infty}\right) \cong \mathscr{G}$.

\section{Acknowledgements}

The authors are grateful to B. Conrad, J. T. Stafford, and M. Van den Bergh for helpful conversations; to S. Kleiman for help with references; and to the referee for several helpful questions and comments.

Nevins was supported by NSF grant DMS-0757987. Sierra was supported by an NSF Postdoctoral Research Fellowship, grant DMS-0802935.

\section{References}

[Artin and Van den Bergh 1990] M. Artin and M. Van den Bergh, "Twisted homogeneous coordinate rings", J. Algebra 133:2 (1990), 249-271. MR 91k:14003 Zbl 0717.14001

[Artin and Zhang 2001] M. Artin and J. J. Zhang, "Abstract Hilbert schemes", Algebr. Represent. Theory 4:4 (2001), 305-394. MR 2002h:16046 Zbl 1030.14003

[Artin et al. 1990] M. Artin, J. Tate, and M. Van den Bergh, "Some algebras associated to automorphisms of elliptic curves", pp. 33-85 in The Grothendieck Festschrift, vol. 1, edited by P. Cartier et al., Progr. Math. 86, Birkhäuser, Boston, MA, 1990. MR 92e:14002 Zbl 0744.14024

[Artin et al. 1999] M. Artin, L. W. Small, and J. J. Zhang, "Generic flatness for strongly Noetherian algebras”, J. Algebra 221:2 (1999), 579-610. MR 2001a:16006 Zbl 0958.16024

[Van den Bergh 1996] M. Van den Bergh, "A translation principle for the four-dimensional Sklyanin algebras”, J. Algebra 184:2 (1996), 435-490. MR 98a:16047 Zbl 0876.17011

[EGA IV.2 1965] A. Grothendieck, "Éléments de géométrie algébrique, IV: Étude locale des schémas et des morphismes de schémas, II", Inst. Hautes Études Sci. Publ. Math. 24 (1965), 5-231. MR 33 \#7330 Zbl 0135.39701

[EGA IV.3 1966] A. Grothendieck, "Éléments de géométrie algébrique, IV: Étude locale des schémas et des morphismes de schémas, III", Inst. Hautes Études Sci. Publ. Math. 28 (1966), 5-255. MR 36 \#178 Zbl 0144.19904

[EGA IV.4 1967] A. Grothendieck, "Éléments de géométrie algébrique, IV: Étude locale des schémas et des morphismes de schémas, IV", Inst. Hautes Études Sci. Publ. Math. 32 (1967), 5-361. MR 39 \#220 Zbl 0153.22301 
[Eisenbud 1995] D. Eisenbud, Commutative algebra: With a view toward algebraic geometry, Graduate Texts in Mathematics 150, Springer, New York, 1995. MR 97a:13001 Zbl 0819.13001

[Gabriel 1962] P. Gabriel, "Des catégories abéliennes", Bull. Soc. Math. France 90 (1962), 323-448. MR 38 \#1144 Zbl 0201.35602

[Harris 1992] J. Harris, Algebraic geometry: A first course, Graduate Texts in Mathematics 133, Springer, New York, 1992. MR 93j:14001 Zbl 0779.14001

[Hartshorne 1977] R. Hartshorne, Algebraic geometry, Graduate Texts in Mathematics 52, Springer, New York, 1977. MR 57 \#3116 Zbl 0367.14001

[Huybrechts and Lehn 1997] D. Huybrechts and M. Lehn, The geometry of moduli spaces of sheaves, Aspects of Mathematics E31, Friedr. Vieweg \& Sohn, Braunschweig, 1997. MR 98g:14012 Zbl 0872.14002

[Keeler 2000] D. S. Keeler, “Criteria for $\sigma$-ampleness”, J. Amer. Math. Soc. 13:3 (2000), 517-532. MR 2001d:14003 Zbl 0952.14002

[Keeler 2010] D. S. Keeler, “Ample filters and Frobenius amplitude”, J. Algebra 323:10 (2010), 3039-3053. MR 2011f:14029 Zbl 1231.13005

[Keeler et al. 2005] D. S. Keeler, D. Rogalski, and J. T. Stafford, "Naïve noncommutative blowing up", Duke Math. J. 126:3 (2005), 491-546. MR 2006g:14005

[Kleiman 1990] S. L. Kleiman, "Multiple-point formulas, II: The Hilbert scheme", pp. 101-138 in Enumerative geometry (Sitges, 1987), edited by S. Xambó-Descamps, Lecture Notes in Mathematics 1436, Springer, Berlin, 1990. MR 92a:14062

[Laumon and Moret-Bailly 2000] G. Laumon and L. Moret-Bailly, Champs algébriques, Ergeb. Math. Grenzgeb. (3) 39, Springer, Berlin, 2000. MR 2001f:14006 Zbl 0945.14005

[Lazarsfeld 2004] R. Lazarsfeld, Positivity in algebraic geometry, I: Classical setting, line bundles and linear series, Ergeb. Math. Grenzgeb. (3) 48, Springer, Berlin, 2004. MR 2005k:14001a

[Nevins and Sierra 2012] T. A. Nevins and S. J. Sierra, "Canonical birationally commutative factors of noetherian graded algebras", in preparation, 2012.

[Rogalski 2004] D. Rogalski, "Generic noncommutative surfaces", Adv. Math. 184:2 (2004), 289-341. MR 2005e:16047 Zbl 1068.16038

[Rogalski and Stafford 2007] D. Rogalski and J. T. Stafford, "Naïve noncommutative blowups at zero-dimensional schemes”, J. Algebra 318:2 (2007), 794-833. MR 2009a:16055 Zbl 1141.14001

[Rogalski and Stafford 2009] D. Rogalski and J. T. Stafford, "A class of noncommutative projective surfaces”, Proc. Lond. Math. Soc. (3) 99:1 (2009), 100-144. MR 2010j:14007 Zbl 1173.14005

[Rogalski and Zhang 2008] D. Rogalski and J. J. Zhang, "Canonical maps to twisted rings", Math. Z. 259:2 (2008), 433-455. MR 2010b:16056 Zbl 1170.16021

[Sierra 2011] S. J. Sierra, “Geometric idealizer rings”, Trans. Amer. Math. Soc. 363:1 (2011), 457-500. MR 2012c:16085 Zbl 1227.16021

[Stafford and Van den Bergh 2001] J. T. Stafford and M. Van den Bergh, "Noncommutative curves and noncommutative surfaces”, Bull. Amer. Math. Soc. (N.S.) 38:2 (2001), 171-216. MR 2002d:16036 Zbl 1042.16016

[Vistoli 2005] A. Vistoli, "Grothendieck topologies, fibered categories and descent theory", pp. 1-104 in Fundamental algebraic geometry: Grothendieck's FGA explained, Math. Surveys Monogr. 123, American Mathematical Society, Providence, RI, 2005. MR 2223406

Communicated by Michel Van den Bergh

Received 2010-10-28 Revised 2012-04-06 
nevins@illinois.edu

s.sierra@ed.ac.uk
Department of Mathematics, University of Illinois at Urbana-Champaign, 1409 West Green Street, MC-382, Urbana, IL, 61801, United States

School of Mathematics, The University of Edinburgh, James Clerk Maxwell Building, The King's Buildings, Mayfield Road, Edinburgh, EH9 3JZ, United Kingdom http://www.maths.ed.ac.uk/ ssierra/ 


\section{Algebra \& Number Theory}

msp.org/ant

\section{EDITORS}

MANAGING EDITOR

Bjorn Poonen

Massachusetts Institute of Technology

Cambridge, USA

\author{
EDITORIAL BOARD CHAIR \\ David Eisenbud \\ University of California \\ Berkeley, USA
}

\section{BOARD OF EDITORS}

Georgia Benkart

Dave Benson

Richard E. Borcherds

John H. Coates

J-L. Colliot-Thélène

Brian D. Conrad

Hélène Esnault

Hubert Flenner

Edward Frenkel

Andrew Granville

Joseph Gubeladze

Ehud Hrushovski

Craig Huneke

Mikhail Kapranov

Yujiro Kawamata

János Kollár

Yuri Manin

Barry Mazur

Philippe Michel
University of Wisconsin, Madison, USA

University of Aberdeen, Scotland

University of California, Berkeley, USA

University of Cambridge, UK

CNRS, Université Paris-Sud, France

University of Michigan, USA

Freie Universität Berlin, Germany

Ruhr-Universität, Germany

University of California, Berkeley, USA

Université de Montréal, Canada

San Francisco State University, USA

Hebrew University, Israel

University of Virginia, USA

Yale University, USA

University of Tokyo, Japan

Princeton University, USA

Northwestern University, USA

Harvard University, USA

École Polytechnique Fédérale de Lausanne
Susan Montgomery

Shigefumi Mori

Raman Parimala

Jonathan Pila

Victor Reiner

Karl Rubin

Peter Sarnak

Joseph H. Silverman

Michael Singer

Vasudevan Srinivas

J. Toby Stafford

Bernd Sturmfels

Richard Taylor

Ravi Vakil

Michel van den Bergh

Marie-France Vignéras

Kei-Ichi Watanabe

Efim Zelmanov
University of Southern California, USA

RIMS, Kyoto University, Japan

Emory University, USA

University of Oxford, UK

University of Minnesota, USA

University of California, Irvine, USA

Princeton University, USA

Brown University, USA

North Carolina State University, USA

Tata Inst. of Fund. Research, India

University of Michigan, USA

University of California, Berkeley, USA

Harvard University, USA

Stanford University, USA

Hasselt University, Belgium

Université Paris VII, France

Nihon University, Japan

University of California, San Diego, USA

\section{PRODUCTION}

production@msp.org

Silvio Levy, Scientific Editor

See inside back cover or msp.org/ant for submission instructions.

The subscription price for 2013 is US \$200/year for the electronic version, and \$350/year ( $\$ 40$, if shipping outside the US) for print and electronic. Subscriptions, requests for back issues and changes of subscribers address should be sent to MSP.

Algebra \& Number Theory (ISSN 1944-7833 electronic, 1937-0652 printed) at Mathematical Sciences Publishers, 798 Evans Hall \#3840, c/o University of California, Berkeley, CA 94720-3840 is published continuously online. Periodical rate postage paid at Berkeley, CA 94704, and additional mailing offices.

ANT peer review and production are managed by EditFLOW ${ }^{\circledR}$ from Mathematical Sciences Publishers.

\section{PUBLISHED BY}

- mathematical sciences publishers

nonprofit scientific publishing

http://msp.org/

(C) 2013 Mathematical Sciences Publishers 


\section{Algebra \& Number Theory}

$\begin{array}{lll}\text { Volume } 7 & \text { No. } 4 \quad 2013\end{array}$

Explicit Chabauty over number fields

SAMIR SIKSEK

Moduli spaces for point modules on naïve blowups

Thomas A. Nevins and Susan J. Sierra

Density of rational points on certain surfaces

Sir Peter SWInNerton-Dyer

HENRIK RUSSELL

Chai's conjecture and Fubini properties of dimensional motivic integration

RAF Cluckers, François LoESER and JohanNes NiCAISE

SHUNSUKE TAKAGI

Finitely presented exponential fields

JONATHAN KIRBY

On a problem of Arnold: The average multiplicative order of a given integer

PÄr Kurlberg and CARL POMERANCE

An analogue of Sturm's theorem for Hilbert modular forms 